\title{
METAL-POOR LITHIUM-RICH GIANTS IN THE RADIAL VELOCITY EXPERIMENT SURVEY*
}

\author{
Gregory R. Ruchtit ${ }^{1,20}$, Jon P. Fulbright ${ }^{1}$, Rosemary F. G. Wyse ${ }^{1}$, Gerard F. Gilmore ${ }^{2,3}$, Eva K. Grebel ${ }^{4}$, \\ Olivier Bienaymé ${ }^{5}$, Joss Bland-Hawthorn ${ }^{6}$, Ken C. Freeman ${ }^{7}$, Brad K. Gibson ${ }^{8,9}$, Ulisse Munari ${ }^{10}$, Julio F. Navarro ${ }^{11}$, \\ Quentin A. Parker ${ }^{12,13,14}$, Warren Reid ${ }^{13}$, George M. Seabroke ${ }^{15}$, Arnaud Siebert $^{5}$, Alessandro Siviero ${ }^{16,17}$, \\ Matthias Steinmetz ${ }^{17}$, Fred G. Watson ${ }^{12}$, Mary Williams ${ }^{17}$, and Tomaz Zwitter ${ }^{18,19}$ \\ ${ }^{1}$ Bloomberg Center for Physics \& Astronomy, Johns Hopkins University, 3400 North Charles Street, Baltimore, MD 21218, USA: gruchti@ mpa-garching.mpg.de \\ ${ }^{2}$ Institute of Astronomy, University of Cambridge, Madingley Road, Cambridge CB3 OHA, UK \\ ${ }^{3}$ Astronomy Department, Faculty of Science, King Abdulaziz University, P.O. Box 80203, Jeddah 21589, Saudi Arabia \\ ${ }^{4}$ Astronomisches Rechen-Institut, Zentrum für Astronomie der Universität Heidelberg, Mönchhofstr. 12-14, D-69120 Heidelberg, Germany \\ ${ }_{5}$ Observatoire de Strasbourg, 11 Rue de l'Université, F-67000 Strasbourg, France \\ ${ }^{6}$ Sydney Institute for Astronomy, School of Physics A28, University of Sydney, NSW 2006, Australia \\ ${ }^{7}$ RSAA Australian National University, Mount Stromlo Observatory, Cotter Road, Weston Creek, Canberra, ACT 2611, Australia \\ ${ }^{8}$ Jeremiah Horrocks Institute for Astrophysics \& Super-computing, University of Central Lancashire, Preston PR1 2HE, UK \\ ${ }^{9}$ Department of Astronomy \& Physics, Saint Marys University, Halifax B3H 3C3, Canada \\ ${ }^{10}$ INAF Osservatorio Astronomico di Padova, Via dell'Osservatorio 8, I-36012 Asiago, Italy \\ ${ }^{11}$ Department of Physics and Astronomy, University of Victoria, P.O. Box 3055, Station CSC, Victoria, BC V8W 3P6, Canada \\ 12 Australian Astronomical Observatory, Coonabarabran, NSW 2357, Australia \\ ${ }^{13}$ Department of Physics and Astronomy, Macquarie University, Sydney, NSW 2109, Australia \\ ${ }^{14}$ Macquarie Research Centre for Astronomy, Astrophysics \& Astrophotonics, Macquarie University, Sydney, NSW 2109, Australia \\ ${ }^{15}$ Mullard Space Science Laboratory, University College London, Holmbury St. Mary, Dorking RH5 6NT, UK \\ ${ }^{16}$ Department of Astronomy, Padova University, Vicolo dell'Osservatorio 2, I-35122 Padova, Italy \\ ${ }^{17}$ Leibniz-Institut für Astrophysik Potsdam (AIP), An der Sternwarte 16, D-14482 Potsdam, Germany \\ ${ }^{18}$ Faculty of Mathematics and Physics, University of Ljubljana, Jadranska 19, SK-1000 Ljubljana, Slovenia \\ ${ }_{19}$ Center of Excellence SPACE-SI, Askerceva cesta 12, SK-1000 Ljubljana, Slovenia \\ Received 2011 September 9; accepted 2011 November 7; published 2011 November 29
}

\begin{abstract}
We report the discovery of eight lithium-rich field giants found in a high-resolution spectroscopic sample of over 700 metal-poor stars $([\mathrm{Fe} / \mathrm{H}]<-0.5)$ selected from the Radial Velocity Experiment survey. The majority of the Li-rich giants in our sample are very metal-poor $([\mathrm{Fe} / \mathrm{H}] \lesssim-1.9)$, and have a $\mathrm{Li}$ abundance (in the form of ${ }^{7} \mathrm{Li}$ ), $A(\mathrm{Li})=\log (n(\mathrm{Li}) / n(\mathrm{H}))+12$, between 2.30 and 3.63, well above the typical upper red giant branch (RGB) limit, $A(\mathrm{Li})<0.5$, while two stars, with $A(\mathrm{Li}) \sim 1.7-1.8$, show similar lithium abundances to normal giants at the same gravity. We further included two metal-poor, Li-rich globular cluster giants in our sample, namely the previously discovered M3-IV101 and newly discovered (in this work) M68-A96. This comprises the largest sample of metal-poor Li-rich giants to date. We performed a detailed abundance analysis of all stars, finding that the majority of our sample stars have elemental abundances similar to that of Li-normal halo giants. Although the evolutionary phase of each Li-rich giant cannot be definitively determined, the Li-rich phase is likely connected to extra mixing at the RGB bump or early asymptotic giant branch that triggers cool bottom processing in which the bottom of the outer convective envelope is connected to the H-burning shell in the star. The surface of a star becomes Li-enhanced as ${ }^{7} \mathrm{Be}$ (which burns to ${ }^{7} \mathrm{Li}$ ) is transported to the stellar surface via the Cameron-Fowler mechanism. We discuss and discriminate among several models for the extra mixing that can cause Li production, given the detailed abundances of the Li-rich giants in our sample.
\end{abstract}

Key words: globular clusters: individual (M68, M3) - stars: abundances - stars: late-type - stars: Population II

Online-only material: color figures

\section{INTRODUCTION}

Lithium (Li) plays a special role in our understanding of the universe. $\mathrm{Li}$, in the form of ${ }^{7} \mathrm{Li}$, is one of four isotopes synthesized immediately after the big bang (Steigman 2007). The latest estimate of the cosmic baryon density from Wilkinson Microwave Anisotropy Probe, WMAP, $\Omega_{b} h^{2}=0.02273 \pm$ 0.00062 (Dunkley et al. 2009), implies a primordial abundance ${ }^{21}$ of $A(\mathrm{Li})=2.72 \pm 0.06$, using the updated reaction rates for ${ }^{3} \mathrm{He}(\alpha, \gamma){ }^{7} \mathrm{Li}$ in the standard big bang nucleosynthesis calculations (Cyburt et al. 2008). This value of the primordial

\footnotetext{
* Based on observations taken at the Keck, Apache Point, Las Campanas, and La Silla (ESO proposal ID: 082.B-0484) Observatories.

${ }^{20}$ Current address: Max Planck Institut für Astrophysik, Postfach 1317,

Karl-Schwarzschild-Str. 1, D-85748 Garching, Germany.

$21 \quad A(\mathrm{Li})=\log (n(\mathrm{Li}) / n(\mathrm{H}))+12$.
}

lithium-7 abundance is significantly higher than that derived for metal-poor stars, for which $A(\mathrm{Li})=2.0-2.4$ for $[\mathrm{Fe} / \mathrm{H}]=$ -3.5 to -1.0 dex (Spite \& Spite 1982a, 1982b; Ryan et al. 2001; Meléndez \& Ramírez 2004; Charbonnel \& Primas 2005; Asplund et al. 2006; Bonifacio et al. 2007; Aoki et al. 2009; Hosford et al. 2009; Sbordone et al. 2010). Lithium is expected to be destroyed in stars, creating helium, in regions where the temperature exceeds a few times $10^{6} \mathrm{~K}$. However, the large amplitude of the discrepancy with the predicted primordial value of lithium-7, together with its apparent constancy over a range of stellar effective temperatures and gravities (the "Spite Plateau"), has stimulated much interest into both lithium destruction and production in stellar interiors and into extensions of the Standard Model of particle physics.

The abundance of $\mathrm{Li}$ in stellar atmospheres is a very useful probe of the structure of the stellar interior and the physical 
processes taking place there. The fragile Li nucleus is readily destroyed when the material is exposed to temperatures exceeding $2.6 \times 10^{6} \mathrm{~K}$, so that strong Li depletion is usually observed in any star whose surface convection zone extends deep enough. As soon as a star moves beyond the sub-giant branch, convective depletion brings $A(\mathrm{Li})$ down by more than one order of magnitude (Pilachowski et al. 1993; Gratton et al. 2000), and $A(\mathrm{Li})<0.5$ is typical for stars on the upper red giant branch (RGB; Lind et al. 2009b). Any giant with a Li abundance above this value is considered Li-rich. About $1 \%$ of solar-metallicity giant stars show large Li abundances (Brown et al. 1989). These giants pose a serious problem for standard stellar evolution models and have triggered a widespread interest in $\mathrm{Li}$ production in giant stars.

Standard models of stellar evolution predict that Li can be produced in the interior, but it is immediately destroyed by nuclear burning, as explained above. Further, pre-existing Li in the surface layers of a star is burned away due to dilution. However, should there exist efficient, extra mixing between the surface and the Li-forming layers, ${ }^{7} \mathrm{Li}$ (or its progenitor ${ }^{7} \mathrm{Be}$ ) can be brought to the cool layers before it burns. Many have tried to explain these phenomena using both internal and external processes.

Cameron (1955) and Cameron \& Fowler (1971) first proposed a mechanism in which Li could be produced (and survive) by ${ }^{7} \mathrm{Be}$ transport to the surface of intermediate-mass asymptotic giant branch (AGB) stars, known as the Cameron-Fowler mechanism. At this stage the outer convective envelope is in contact with the H-burning shell where ${ }^{3} \mathrm{He}$ enrichment has taken place from the proton-proton reaction chain. The ${ }^{3} \mathrm{He}$ is transported to regions with temperatures high enough to burn it to ${ }^{7} \mathrm{Be}$ by the ${ }^{3} \mathrm{He}(\alpha, \gamma){ }^{7} \mathrm{Be}$ reaction. The ${ }^{7} \mathrm{Be}$ is then swept up to the stellar surface where it decays to ${ }^{7} \mathrm{Li}$ by electron captures $\left({ }^{7} \mathrm{Be}\left(e^{-}, v\right)^{7} \mathrm{Li}\right)$. This process of ${ }^{3} \mathrm{He}$ transport from the inner $\mathrm{H}$-burning shell and subsequent burning to ${ }^{7} \mathrm{Be}$ is otherwise known as "hot bottom burning" (cf. Forestini \& Charbonnel 1997, and references therein).

Later, Sackmann \& Boothroyd (1999) showed that the Cameron-Fowler mechanism can also occur for low-mass giants evolving on the RGB due to extra deep mixing and "cool bottom processing" (CBP), in which material from the cool bottom of the outer convective envelope reaches temperatures in which the ${ }^{3} \mathrm{He}$ is burned. This process was first postulated to explain the abundances of ${ }^{13} \mathrm{C}$ in AGB and RGB stars (Boothroyd et al. 1995). To produce enhanced ${ }^{7} \mathrm{Li}$ on the surface, CBP requires high mixing rates $\left(\dot{M}_{p} \gtrsim 10^{-7} M_{\odot} \mathrm{yr}^{-1}\right)$. Depending on this mixing rate, a star can achieve a Li enhancement upward of $A(\mathrm{Li}) \sim 4$. Sackmann \& Boothroyd (1999) suggest that the rarity of Li-rich giants implies that few stars can achieve high enough mixing rates to drive $\mathrm{Li}$ production or that the episode of rapid mixing is brief. Boothroyd \& Sackmann (1999) also note that low-mass metal-poor RGB stars should undergo more aggressive CBP than metal-rich giants, since the extra mixing will reach higher temperatures. Further, if the ${ }^{3} \mathrm{He}$ is not fully depleted during the evolution up the RGB, then CBP can also occur on the AGB. In low-mass AGB stars, the outer envelope and H-burning shell are not in contact. Deep extra mixing via $\mathrm{CBP}$ can connect the two regions, allowing the Cameron-Fowler mechanism to occur in low-mass AGB stars (Nollett et al. 2003).

More recently, thermohaline mixing (Charbonnel \& Primas 2005; Charbonnel \& Zahn 2007) and magneto-thermohaline mixing (Denissenkov et al. 2009) have been proposed as sources of extra mixing to drive Li production on the surface layers of stars. On the other hand, extra mixing processes at the RGB bump (Charbonnel 1995) may also induce a so-called Li flash (Palacios et al. 2001). In this case, ${ }^{7} \mathrm{Be}$ is transported to the surface layers of a star, while the star is also rapidly increasing in luminosity. This model was later challenged by Denissenkov \& Herwig (2004), who independently found that canonical extra mixing in stars cannot produce a Li flash. In their more recent investigations, Palacios et al. (2006) were still not able to validate the Li-flash model.

Another scenario invokes mass-loss mechanisms on the RGB, which are accompanied by extra mixing that increases the Li abundance (De la Reza et al. 1996, 2000). Note, however, that subsequent searches (e.g., Fekel \& Watson 1998; Jasniewicz et al. 1999) did not detect any Li-rich stars in samples of giants with far-infrared excess. Finally, external angular momentum from a companion object (brown dwarf or giant planet) may induce the extra mixing needed to drive the Cameron-Fowler mechanism (Denissenkov \& Herwig 2004), which in turn leads to increased $\mathrm{Li}$.

These models are described in detail in Section 5, a but common feature among these theories is that the Li enrichment on the surface layers of a star can reach values $\sim 2$ dex higher than the Spite Plateau. Further, the Li-enrichment phase is short, lasting about 2 Myr (see Section 5 and, e.g., Denissenkov \& Herwig 2004). Another important aspect of these models is that material from the CNO-burning regions is also transported to the stellar surface. The distinguishing features among the models include the mixing mechanism and the timing of the mixing/burning episodes along a star's evolution, which can affect the amount of $\mathrm{CNO}$ material brought to the stellar surface. These could also have an effect on the abundances of heavier elements, such as $r-, s$-, and possibly $p$-process elements present in the star's atmosphere. Detailed elemental abundances of Li-rich giants will therefore provide insight into mixing and nucleosynthesis processes within evolved stars, and will ultimately further our understanding of the origins of these peculiar stars.

Li-rich giants have been discovered in the field (Charbonnel \& Balachandran 2000; Roederer et al. 2008; Kumar \& Reddy 2009; Kumar et al. 2011; Monaco et al. 2011b) and Galactic bulge (Uttenthaler et al. 2007; Gonzalez et al. 2009), as well as in globular clusters (Carney et al. 1998; Kraft et al. 1999; Smith et al. 1999) and dwarf spheroidal galaxies (Domínguez et al. 2004; Monaco \& Bonifacio 2008). These samples contain stars on both the RGB and AGB that range in mass $\left(\sim 0.8-5 M_{\odot}\right)$ and have metallicities from solar down to $\sim-2$ dex. It is important to note, however, that the vast majority of known Li-rich giants have metallicities near solar, while few $(\sim 4-5)$ Li-rich giants have been discovered with $[\mathrm{Fe} / \mathrm{H}]<-1$.

In their compilation of near solar-metallicity stars, Charbonnel \& Balachandran (2000) found that Li-rich giants primarily cluster around two regions in the color-magnitude diagram. They associated low-mass Li-rich giants with the RGB luminosity bump (RGB bump), while those with intermediate masses were assumed to be evolving on the early AGB. This is convenient, because in both regions extra mixing can be triggered after the molecular weight discontinuity from the first dredge-up is erased (see above). Charbonnel \& Balachandran (2000) suggested that the localization of the Li-rich giants into two groups argued against the interaction with a companion object as the cause for Li production. This two-region picture, however, has been challenged by the discovery of low-mass Li-rich giants that lie near the RGB tip (e.g., Kraft et al. 1999; Monaco \& Bonifacio 2008), where Charbonnel \& Balachandran (2000) 
Table 1

Observational Data

\begin{tabular}{|c|c|c|c|c|c|c|c|}
\hline Star & $\begin{array}{l}\text { R.A. }^{a} \\
\left({ }^{\circ}\right)\end{array}$ & $\begin{array}{c}\text { Decl. }^{\mathrm{a}} \\
\left({ }^{\circ}\right)\end{array}$ & Mag. ${ }^{b}$ & $\begin{array}{c}\text { Obsdate } \\
\text { (yyyymmdd) }\end{array}$ & Observatory $^{\mathrm{c}}$ & Instrument & $\begin{array}{c}\mathrm{S} / \mathrm{N}^{\mathrm{d}} \\
\left(\text { pixel }^{-1}\right)\end{array}$ \\
\hline C1012254-203007 & 153.106 & -20.502 & 11.6 & 20070506 & LCO & MIKE & 100 \\
\hline J043154.1-063210 & 67.976 & -6.536 & 10.4 & 20071219 & APO & ARCES & 100 \\
\hline J142546.2-154629 & 216.443 & -15.775 & 9.8 & 20090212 & La Silla & FEROS & 130 \\
\hline J195244.9-600813 & 298.187 & -60.137 & 10.1 & 20081016 & LCO & MIKE & 160 \\
\hline T5496-00376-1 & 155.025 & -13.095 & 9.1 & 20070421 & APO & ARCES & 130 \\
\hline T6953-00510-1 & 327.969 & -22.935 & 9.9 & 20081016 & LCO & MIKE & 140 \\
\hline T8448-00121-1 & 348.250 & -45.119 & 10.0 & 20070922 & AAT & UCLES & 100 \\
\hline T9112-00430-1 & 312.012 & -65.966 & 10.4 & 20081016 & LCO & MIKE & 140 \\
\hline M3-IV101 & 196.259 & 28.303 & $(13.2)$ & 19990323 & Keck & HIRES & 80 \\
\hline M68-A96 & 180.659 & -26.717 & $(13.0)$ & 20040106 & LCO & du Pont Echelle & 100 \\
\hline
\end{tabular}

Notes.

${ }^{a}$ Equinox 2000

b The I magnitudes from the RAVE database are given here for the eight RAVE stars. $V$ magnitudes are given in parentheses for M3-IV101 (Johnson \& Sandage 1956) and M68-A96 (Alcaino 1977).

c LCO: Los Campanas Observatory; APO: Apache Point Observatory; AAT: Australian Astronomical Telescope.

d Estimated between 5500 and $6000 \AA$.

had classified solar-metallicity stars as intermediate-mass AGB. Further, in a study of Li-rich giants in the thick disk of the Milky Way, Monaco et al. (2011b) found that the Li-rich giants in their sample did not fall in either group defined by Charbonnel \& Balachandran (2000).

Metal-poor low-mass Li-rich giants near the RGB tip further confuse the situation. It is possible that these stars have not yet reached the AGB. Indeed, Kraft et al. (1999) found that the Li-rich giant, M3-IV101, had a luminosity placing it near the RGB tip, but was most likely an RGB star according to the color-magnitude diagram of $\mathrm{M} 3$, using high-precision photometry. How can we distinguish between the RGB and AGB for metal-poor stars? A possible discriminant (in addition to the $\mathrm{CNO}$ abundance differences between different theories of Li production) is that AGB stars that have begun the third dredge-up typically show enhancements in the $s$-process elements as compared to RGB stars. As stated above, the number of known metal-poor Li-rich giants is quite small. A clear classification of the evolutionary phase of these stars is absolutely critical for understanding the processes that create the Lithium. It is therefore important to identify and analyze more metal-poor Li-rich giants.

We have discovered nine candidate Li-rich metal-poor giants. Eight of the stars were part of high-resolution observations of metal-poor stars selected from the Radial Velocity Experiment survey (RAVE; Steinmetz et al. 2006) and one, in the very metal-poor (VMP) globular cluster M68, was found by us independently of RAVE. In this paper, we report on the abundance properties of these stars and investigate possible signatures for each star's evolutionary stage, and look for supporting evidence of the mechanism for enhanced Li production.

\section{OBSERVATIONS}

The Li-rich stars reported here were among over 700 candidate metal-poor stars selected for high-resolution observations (Ruchti et al. 2010; Fulbright et al. 2010), based on data obtained by the RAVE survey, with the exception of M68-A96, whose Li-rich nature was discovered during observations of stars in that globular cluster. The full details of the high-resolution observations and reductions of the RAVE stars can be found in the papers cited above, but some information is given in Table 1. M68-A96 was observed with the echelle spectrograph on the Irénée du Pont $2.5 \mathrm{~m}$ telescope at the Las Campanas Observatory. For comparison purposes, we also analyzed a blue Keck/HIRES spectrum taken in 1999 March of the previously known Li-rich giant M3 IV-101. Note that this spectrum is not the same as that used by Kraft et al. (1999).

The spectrographs used for our observations deliver a resolving power greater than 30,000. The signal-to-noise ratio $(\mathrm{S} / \mathrm{N})$ levels of the observed spectra are quite good: nearly all have S/Ns greater than 100 pixel $^{-1}$. With the exception of the UCLES spectrum, the wavelength coverage goes from below $4000 \AA$ to beyond $8000 \AA$ (for UCLES the range is roughly $4460-7260 \AA$ ), although there are some gaps in coverage. In each case the data were reduced using standard reduction methods for echelle data, utilizing pipeline reduction programs when available.

During routine inspection of the $\sim 700$ spectra taken, we noticed that the $6708 \AA$ Li I lines in some of the stars' spectra were unusually strong. For example, the Li I line in the star J142546.2-154629 has an equivalent width (EW) of $\sim 540 \mathrm{~m} \AA$ (see Section 4.1). This is roughly twice as strong as each of the $\mathrm{Na} D$ lines in this star. In several of the other spectra, the $6708 \AA$ line appeared on two adjacent orders, so it was very unlikely that the feature was an artifact introduced by some feature of the observation or reductions. The $6103 \AA \mathrm{Li}$ I line was also visible in most of these anomalous stars, confirming the high $\mathrm{Li}$ abundances.

\section{ANALYSIS}

\subsection{Stellar Parameters}

The abundance determinations were achieved with the MOOG analysis program (Sneden 1973), using onedimensional, plane-parallel Kurucz model atmospheres ${ }^{22}$ under the assumption of static equilibrium and LTE. Stellar parameters were derived following a variation of the methods described in Ruchti et al. (2010, 2011). The initial effective temperature, $T_{\mathrm{ini}}$, was set by using the excitation temperature method based on $\mathrm{Fe}$ I lines. The initial value of the surface gravity $\left(\log g_{\text {ini }}\right)$ was set using the ionization equilibrium criterium utilizing the

\footnotetext{
22 See http://kurucz.harvard.edu/
} 
Table 2

Stellar Parameter Values

\begin{tabular}{|c|c|c|c|c|c|c|}
\hline (error) & $\begin{array}{l}T_{\text {eff }} \\
(\mathrm{K}) \\
\pm 140\end{array}$ & \pm 0.3 & \pm 0.10 & $\begin{array}{c}v_{t} \\
\left(\mathrm{~km} \mathrm{~s}^{-1}\right) \\
\pm 0.1\end{array}$ & $\begin{array}{c}\log \left(L / L_{\odot}\right) \\
\quad \pm 0.3\end{array}$ & $\mathrm{POP}^{\mathrm{a}}$ \\
\hline C1012254-203007 & 4518 & 1.1 & -2.55 & 2.2 & 2.9 & 3 \\
\hline J043154.1-063210 & 5440 & 2.5 & -1.85 & 1.3 & 1.8 & 3 \\
\hline J142546.2-154629 & 4341 & 0.9 & -2.08 & 2.3 & 3.0 & 3 \\
\hline J195244.9-600813 & 5025 & 2.1 & -1.41 & 1.7 & 2.0 & 2.5 \\
\hline T5496-00376-1 & 4887 & 2.2 & -0.63 & 1.4 & 1.9 & 2 \\
\hline Т6953-00510-1 & 4867 & 1.8 & -1.93 & 1.8 & 2.3 & 2 \\
\hline T8448-00121-1 & 4655 & 1.1 & -2.45 & 2.0 & 2.9 & 3 \\
\hline T9112-00430-1 & 4370 & 0.0 & -2.21 & 3.1 & 3.5 & 3 \\
\hline M3-IV101 & 4236 & 0.6 & -1.52 & 1.8 & 3.2 & - \\
\hline M68-A96 & 4549 & 0.9 & -2.18 & 1.8 & 3.1 & - \\
\hline
\end{tabular}

Note. ${ }^{\text {a }}$ 2: thick disk; 2.5 : thick/halo; 3 : halo.

iron abundance derived by both Fe I and Fe II lines. The initial $[\mathrm{Fe} / \mathrm{H}]$ value of the stellar atmosphere for each star was chosen to match the $[\mathrm{Fe} I \mathrm{II} / \mathrm{H}]$ value derived from the analysis. The value of the microturbulent velocity $\left(v_{t}\right)$ was set to minimize the magnitude of the slope of the relationship between the iron abundance derived from $\mathrm{Fe}$ I lines and the value of the reduced width of the line.

In Ruchti et al. (2010, 2011), we found during our analysis of the spectra for several globular cluster giants and giant stars selected from the Fulbright (2000) sample that the effective temperature estimate from the excitation method showed an offset that correlated with $[\mathrm{Fe} / \mathrm{H}]_{\text {ini }}$, when compared to photometric temperature estimates (using the Two Micron All Sky Survey, 2MASS, color-temperature transformations of González Hernández \& Bonifacio 2009). We have since found similar results using the color-temperature transformations of L. Casagrande et al. (2011, private communication). We therefore applied the temperature corrections described in Ruchti et al. (2011) to the Li-rich candidates in our current sample. The analysis described above was then performed again, but with the effective temperature now forced to equal the corrected temperature estimate, $T_{\text {eff }}$. The final adopted values of the stellar parameters for our Li-rich stars are given in Table 2. The error in effective temperature, $\sigma_{T_{\text {eff }}}=140 \mathrm{~K}$, and $[\mathrm{Fe} / \mathrm{H}], \sigma_{[\mathrm{Fe} / \mathrm{H}]}=0.10$ dex, were adopted from Ruchti et al. $(2010,2011)$. We estimated an error in surface gravity of 0.3 dex; however, the error could be larger for the lowest gravity stars (as is described below).

The method of using ionization equilibrium to derive the surface gravity is believed to be unreliable in VMP stars due to non-LTE effects (Thevenin \& Idiart 1999; Kraft \& Ivans 2003). The abundance derived from the Li I line, however, is nearly independent of the adopted surface gravity. In Ruchti et al. $(2010,2011)$, it was assumed that no giants lie above the RGB tip, but we do not make this assumption here since the evolutionary stage of our giants will affect the interpretation of our abundance results. We therefore did not apply any of the corrections described in Ruchti et al. (2011) to the surface gravity of our Li-rich candidates. Most of our Li-rich candidates have $\log g>1.0$, values which were not corrected in Ruchti et al. $(2010,2011)$. Three stars have $\log g=0.6-0.8$, for which the correction would only be $0.1-0.2$ dex, well within our errors. The star T9112-00430-1 has the lowest gravity, suggesting it lies far above the RGB tip (see Figure 1). The derived values of the stellar parameters (specifically gravity) of this star are most

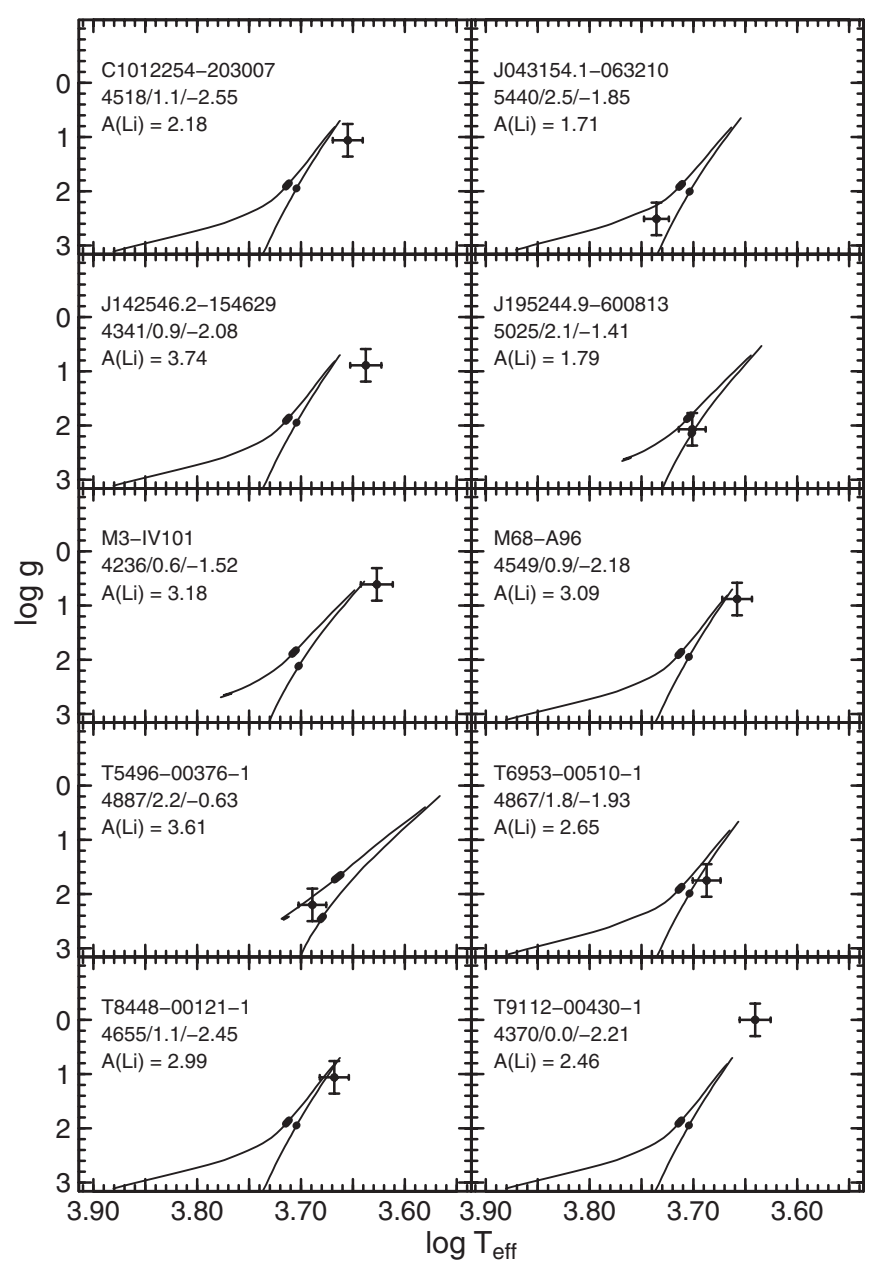

Figure 1. Position of our Li-rich stars in the gravity-temperature plane. Also plotted are 12 Gyr Padova isochrones (Marigo et al. 2008; Girardi et al. 2010) of an appropriate metallicity to that of each star. The name, stellar parameters (given as $T_{\mathrm{eff}} / \log g /[\mathrm{Fe} / \mathrm{H}]$ ), and $\mathrm{Li}$ abundance, $A(\mathrm{Li})$, are shown in each panel. The circle on the RGB and thickened region on the AGB at $\log g \sim 2$ represent the luminosity bumps on the RGB and AGB, respectively. Note that with the values estimated here, T9112-00430-1 lies well above the isochrone, which is most likely a reflection of the inappropriateness, for this star, of the assumption of a steady-state atmosphere in LTE.

likely affected by large non-LTE effects. If we were to increase its gravity estimate by 0.5 dex, as prescribed by Ruchti et al. (2011), it would lie close to the RGB tip.

The majority of our Li-rich candidates have $[\mathrm{Fe} / \mathrm{H}]<-1.8$. The most metal-rich star, T5496-00376- 1 , has $[\mathrm{Fe} / \mathrm{H}]=-0.63$, which is much more metal-rich than the rest of the candidates but is at the lower end of most previous studies. We include it here because of its Li-rich nature. Our estimates of $T_{\text {eff }}$ and $[\mathrm{Fe} / \mathrm{H}]$ for M3-IV101 strongly resemble those found by Kraft et al. (1999), showing differences of only $36 \mathrm{~K}$ and 0.02 dex in $T_{\text {eff }}$ and $[\mathrm{Fe} / \mathrm{H}]$, respectively. Our $\log g$ value, however, is about 0.2 dex lower than their value. This offset is very similar to the correction that would be made to our $\log g$ value if we were to follow the analysis in Ruchti et al. (2011). Our [Fe/H] estimate for M68-A96 also agrees within $\sim 0.02$ dex with metallicity estimates for the M68 cluster (Lee et al. 2005).

\subsection{Luminosity}

The luminosity of each star was estimated by fitting to Padova isochrones (Marigo et al. 2008; Girardi et al. 2010). The $Z$-metallicity of each star was derived by combining $[\mathrm{Fe} / \mathrm{H}]$ and 
Table 3

Lithium and CNO Abundance Data

\begin{tabular}{|c|c|c|c|c|c|c|c|c|c|c|c|c|}
\hline \multirow[t]{2}{*}{ Star } & \multirow[t]{2}{*}[\mathrm{Fe}/\mathrm{H}]{} & \multirow[t]{2}{*}[\mathrm{C}/\mathrm{Fe}]{$^{\mathrm{a}}$} & \multirow[t]{2}{*}[\mathrm{N}/\mathrm{Fe}]{$^{\mathrm{a}}$} & \multirow[t]{2}{*}[\mathrm{O}/\mathrm{Fe}]{$^{\mathrm{a}}$} & \multirow[t]{2}{*}{$\log (\mathrm{C} / \mathrm{O})$} & \multirow[t]{2}{*}{${ }^{12} \mathrm{C} /{ }^{13} \mathrm{C}^{\mathrm{b}}$} & \multicolumn{3}{|c|}{ Li-6708 Å } & \multicolumn{3}{|c|}{ Li-6103 } \\
\hline & & & & & & & EW Li & $A(\mathrm{Li})$ & $A(\mathrm{Li})_{\text {NLTE }}$ & EW Li & $A(\mathrm{Li})$ & $A(\mathrm{Li})_{\mathrm{NLTE}}$ \\
\hline C1012254-203007 & -2.55 & -0.7 & 0.7 & 0.61 & -1.6 & $1-5(1)$ & 279.6 & 2.52 & 2.30 & 6.3 & 1.93 & 2.06 \\
\hline J043154.1-063210 & -1.85 & 0.3 & 0.3 & 0.52 & -0.5 & $5-15(12)$ & 30.0 & 1.69 & 1.71 & - & - & - \\
\hline J142546.2-154629 & -2.08 & 0.0 & 0.6 & 0.72 & -1.0 & $>10(22)$ & 540.0 & 3.86 & 3.63 & 211.6 & 3.85 & 3.85 \\
\hline J195244.9-600813 & -1.41 & -0.6 & 1.1 & 0.53 & -1.4 & $\gtrsim 5(5)$ & 72.7 & 1.73 & 1.79 & - & - & - \\
\hline T5496-00376-1 & -0.63 & -0.3 & 0.4 & 0.34 & -0.9 & $5-20(15)$ & 323.7 & 3.28 & 2.94 & 34.0 & 2.91 & 3.03 \\
\hline T6953-00510-1 & -1.93 & 0.1 & 1.9 & 1.00 & -1.2 & $3-7(5)$ & 254.3 & 2.82 & 2.53 & 8.8 & 2.28 & 2.38 \\
\hline Т8448-00121-1 & -2.45 & -0.3 & 0.4 & 0.14 & -0.7 & $>10(28)$ & 389.2 & 3.71 & 3.06 & 67.5 & 3.19 & 3.29 \\
\hline T9112-00430-1 ${ }^{\mathrm{c}}$ & -2.21 & -0.7 & 0.8 & 0.44 & -1.4 & $>5(10)$ & 485.2 & 3.15 & 3.08 & 68.0 & 2.92 & 3.09 \\
\hline M3-IV101 & -1.52 & -0.5 & 1.2 & 0.24 & -1.0 & $10-20(12)$ & 508.5 & 3.49 & 3.46 & 211.6 & 3.68 & 3.76 \\
\hline M68-A96 & -2.18 & -0.4 & 0.4 & 0.30 & -1.0 & $3-5(4)$ & 336.5 & 2.95 & 2.64 & 23.0 & 2.52 & 2.66 \\
\hline
\end{tabular}

Notes.

a Solar abundances adopted from Asplund et al. (2009).

b Number in parentheses is the best-fit value for $\mathrm{CH}$ and $\mathrm{CN}$ lines between 4200 and $4220 \AA$.

c $A(\mathrm{Li})$ computed for $\log g=0.5$.

alpha-enhancement using the transformation of Salaris et al. (1993). We assumed an alpha-enhancement equal to $[\mathrm{Mg} / \mathrm{Fe}]$ measured for each star (see Section 4.3). We then fit each star to the isochrone with the closest matching metallicity in a grid of $12 \mathrm{Gyr}$ isochrones with metallicity steps of $Z=0.00002$. It is possible that some of our stars are younger (especially T549600376-1), but the error in the luminosity due to our uncertainty in $\log g$ far outweighs this. Figure 1 shows each star and the isochrone of the same Z-metallicity in the gravity-temperature plane.

The luminosity at the point on the isochrone with the same $\log g$ value as the star being fitted was chosen as the luminosity of the star, except for T9112-00430-1. This star has a gravity above the limits of the isochrone (see Figure 1). We therefore adopted the luminosity at the point of lowest gravity on the isochrone. We linearly interpolated the luminosity values versus $\log g$ for stars with $\log g$ values lying between the points on the corresponding isochrone. We further estimated two extreme luminosity values for each star by adding and subtracting $0.3 \mathrm{dex}$ to each star's $\log g$ value and then fitting again. Errors were then estimated by taking the difference between these extreme values and the value taken from the original fit. This resulted in a typical error in the luminosity of each star of $\sigma_{\log \left(L / L_{\odot}\right)} \sim 0.3$. Most stars lie between the RGB and AGB branches of the isochrones, making it difficult to label them as one or the other from inspection of Figure 1 alone. The estimated luminosity from fitting to either branch showed no differences. We discuss the phase of evolution of each star in more detail later (see Section 5). Our values for the luminosity of each star are listed in Table 2.

Given these luminosities, we followed the methodology described in Ruchti et al. (2011) to determine in which Galactic population each of the Li-rich giants most likely belongs. The final population assignment for each Li-rich candidate is given in Table 2. Note that the same population assignment was found using the stellar parameters derived in this work for the four stars (J142546.2-154629, J195244.9-600813, T5496-00376-1, and T6953-00510-1) that were also analyzed in Ruchti et al. (2010, 2011). All stars were assigned to the thick disk, halo, or thick/halo intermediate population (see Ruchti et al. 2011 for more details). This implies that the Li-rich giants in our sample are old (>10 Gyr) and metal-poor. Thus, they most likely have low masses, $M<1 M_{\odot}$.

\section{RESULTS}

\subsection{Lithium Abundances}

The Li abundances for each star were derived assuming that all the $\mathrm{Li}$ was from the ${ }^{7} \mathrm{Li}$ isotope. EWs were measured for both the $6708 \AA$ and $6103 \AA \mathrm{Li}$ I lines. We then derived the abundance of Li from both lines in each of LTE and non-LTE following the methods described in Lind et al. (2009a). These values can be found in Table 3.

The derived $A(\mathrm{Li})$ values for our Li-rich sample are shown in Figure 2 as a function of the stars' estimated luminosity (calculated above). Note that, for stars that had $A(\mathrm{Li})$ estimates from both $\mathrm{Li}$ I lines, the value in the figure is the mean of the two values. Further, we found a typical error of $\sim 0.20$ dex from the difference between that found for the $6708 \AA$ and $6103 \AA$ lines. Figure 2 also includes the $\mathrm{Li}$ abundances we derive for a sample of 58 RAVE very metal-poor (hereafter RAVE-VMP) stars with $[\mathrm{Fe} / \mathrm{H}]<-2$ from Fulbright et al. (2010). Note that 26 measurements are only upper limits. We followed the same analysis procedure given above for all RAVE-VMP data, and the full results for the entire sample will be published in a later paper.

Note that both J043154.1-063210 and J195244.9-600813 have luminosities and $\mathrm{Li}$ abundances that place them along the trend of "Li-normal" giants near the RGB bump. We therefore no longer classify them as Li-rich. The remaining Li-rich giants have Li abundances that clearly separate them from the Li-normal giants (see Figure 2). As was found in previous studies, the majority of our Li-rich giants separate into two regions: the lower (near the RGB bump) and upper RGB, separated at $\log \left(L / L_{\odot}\right) \sim 2.4$ (see also Figure 1). T695300510-1, however, appears to lie between these two regions.

\subsection{CNO Abundances}

We determined the CNO abundances for our giants using MOOG, under the assumption of molecular equilibrium, since the CNO atoms can be partly bound together in molecules, especially for cooler stars (see, e.g., Gratton \& Sneden 1990).

Oxygen abundances were first determined from the EWs of the O I forbidden lines at $6300 \AA$ and $6363 \AA$, and are given in Table 3. The $g f$-values of the lines were taken from Lambert (1978), and the solar abundance, $A(\mathrm{O})=8.69$, was selected 


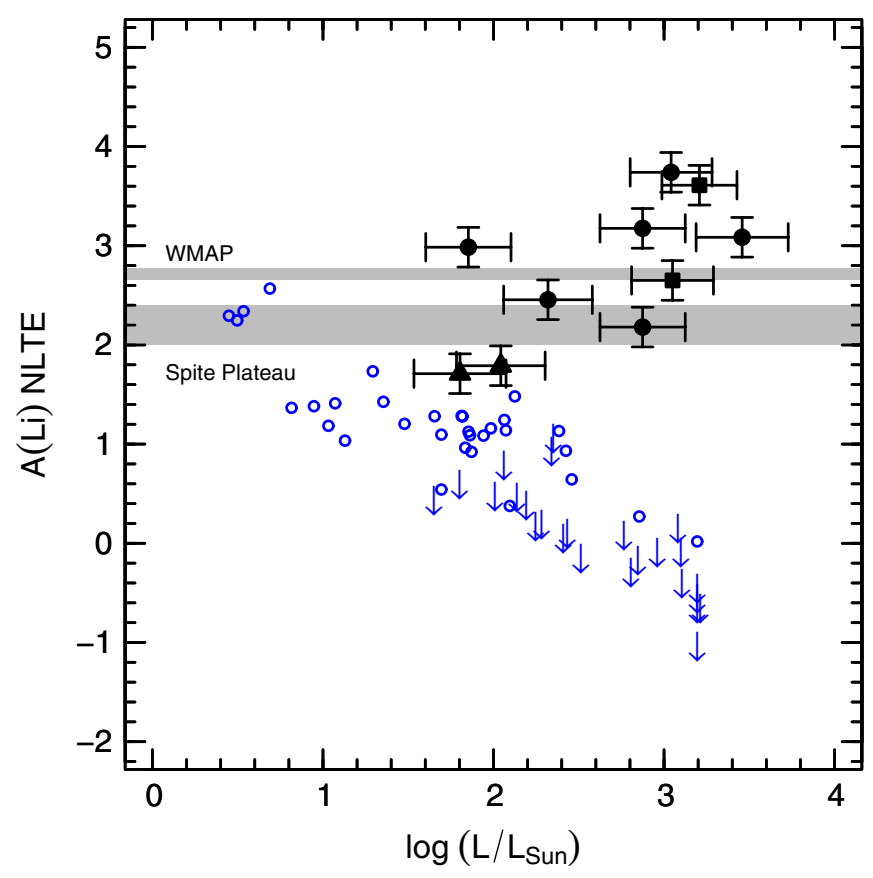

Figure 2. Derived $A(\mathrm{Li})$ abundance as a function of $\log \left(L / L_{\odot}\right)$ for the giants analyzed in this paper. Stars from the RAVE-VMP sample are plotted as blue open circles and downward pointing arrows (for upper limits), and represent "Li-normal" stars. The filled circles represent the six Li-rich field giants discovered in this paper from the RAVE sample, and the filled squares represent the two Li-rich globular cluster giants. The filled triangles represent the two giants in our sample that are more Li-normal for the given luminosity bin. Error bars in $\log \left(L / L_{\odot}\right)$ represent the difference in luminosity when we added or subtracted 0.3 dex from our value of $\log g$ for each star. Also shown are the range of estimates for the primordial abundance of $\mathrm{Li}$ given by WMAP and the Spite Plateau. Note that several of the giants in our sample have derived $\mathrm{Li}$ abundances that are much greater than the primordial values.

(A color version of this figure is available in the online journal.)

from Asplund et al. (2009). We corrected the O I $6300 \AA$ line for the weak Ni I $6300.34 \AA$ line (see Allende Prieto et al. 1999) following the same methodology as Fulbright \& Johnson (2003).

We next determined $[\mathrm{C} / \mathrm{Fe}],[\mathrm{N} / \mathrm{Fe}]$, and the ${ }^{12} \mathrm{C} /{ }^{13} \mathrm{C}$ ratios for our giants by spectral syntheses of $\mathrm{CH}$ and $\mathrm{CN}$ lines using the Plez line lists and oscillator strengths (B. Plez 2011, private communication; see also Hill et al. 2002; Gustafsson et al. 2008) combined with the Vienna Atomic Line Database (VALD; ${ }^{23}$ Kupka et al. 2000). We adopted dissociation energies of $3.47 \mathrm{eV}$ (Huber \& Herzberg 1979) and $7.66 \mathrm{eV}$ (Lambert 1978) for $\mathrm{CH}$ and $\mathrm{CN}$, respectively. The $\mathrm{CH}$ lines between 4320 and $4328 \AA$ were used to estimate the $\mathrm{C}$ abundance of each star given the value of $A(\mathrm{O})$ found above. Given these values of $A(\mathrm{O})$ and $A(\mathrm{C})$, the $\mathrm{N}$ abundance was determined using $\mathrm{CN}$ lines at the $3883 \AA$ band (and the $4216 \AA$ band, see below). Finally, the ${ }^{12} \mathrm{C} /{ }^{13} \mathrm{C}$ isotopic ratio was constrained by combining information from the above lines with that derived from fits to $\mathrm{CH}$ and $\mathrm{CN}$ lines in the wavelength range $4200-4220 \AA$. We then iterated this procedure with the input $\mathrm{CNO}$ abundances equal to the previous iteration until we obtain a self-consistent solution to all three abundances.

During the syntheses of $\mathrm{C}$ and $\mathrm{N}$, the Li-rich candidates fell into two classes: those with a strong CN-3883 band and those with a weak one. The final syntheses are shown in Figures 3 and 4 , respectively. The lines in the $\mathrm{CN}$-strong stars could very well be saturated (most obvious in the M3-IV101 spectrum).

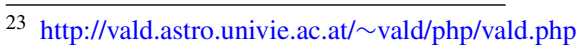

We therefore included the $4216 \AA \mathrm{CN}$ band in our syntheses to constrain the $\mathrm{N}$ abundance for these stars. Although the $\mathrm{CN}-4216$ band is very weak for the $\mathrm{CN}$-weak stars, we include it in Figure 4 for comparison.

The resultant $[\mathrm{C} / \mathrm{Fe}]$ and $[\mathrm{N} / \mathrm{Fe}]$ values from the syntheses are given in Table 3 and plotted versus $\log g$ in Figure 5. Note that we adopted the solar abundances for $\mathrm{C}$ and $\mathrm{N}$ from Asplund et al. (2009). The scatter in individual $[\mathrm{C} / \mathrm{Fe}]$ and $[\mathrm{N} / \mathrm{Fe}]$ values is due to variations between stars, not the quality of the determination (we investigate the interpretation of this scatter in a separate paper). We estimated the internal errors to be $\sim 0.1$ dex in $[\mathrm{C} / \mathrm{Fe}]$ and $\sim 0.1-0.2$ dex in $[\mathrm{N} / \mathrm{Fe}]$, depending on the quality of the spectrum and the strength of the $\mathrm{CN}$ bands. We further varied the estimated values of the stellar parameters of each star according to their $1 \sigma$ errors to investigate the sensitivity of our syntheses to the chosen values of the stellar parameters for each star. Our stars consistently show errors of $\sim 0.1$ and $\sim 0.1-0.2$ dex in $[\mathrm{C} / \mathrm{Fe}]$ and $[\mathrm{N} / \mathrm{Fe}]$, respectively, and show the highest sensitivity to changes in the effective temperature of a star. Using the same error analysis as for $\mathrm{C}$ and $\mathrm{N}$, we found that our errors in $[\mathrm{O} / \mathrm{Fe}]$ are about 0.10 dex. It is important to note, however, that the EWs for C1012252-203007, J043154.1-063210, M68A96, and T8448-00121-1 are less than $10 \mathrm{~m} \AA$ for both lines, and so the error in $[\mathrm{O} / \mathrm{Fe}]$ increases to $\sim 0.15-0.20$ dex. The ${ }^{12} \mathrm{C} /{ }^{13} \mathrm{C}$ isotopic ratio was very difficult to constrain since most of our stars were fairly deficient in carbon (see below) and so the ${ }^{13} \mathrm{CH}$ features were very weak. The best-fit value for most stars subsequently had a fairly flat likelihood peak. We therefore give a range of values, as well as the best-fit value, in Table 3 .

The CNO abundances of M3-IV101 have been analyzed previously in the literature, which makes it useful for comparisons. We only compare the abundance values, since there will be offsets in the element ratios with iron due to differences in reference solar abundances. Kraft et al. (1999) found using $\mathrm{CH}$ lines that $A(\mathrm{C}) \sim 6.55$ for this star, while Pilachowski et al. (2003) found a value of $\sim 6.31$ using $\mathrm{CO}$ lines. Our value of $[\mathrm{C} / \mathrm{Fe}]=-0.5$ corresponds to $A(\mathrm{C})=6.4$, which lies between the Kraft et al. and Pilachowski et al. values. The variation in the values could be attributed to differences in atomic and molecular line data. On the other hand, we found values of $A(\mathrm{O})=7.41$ and $A(\mathrm{~N})=7.61$, which are only 0.01 dex and 0.06 dex higher than those found by Kraft et al. (1999), respectively. Note that Pilachowski et al. (2003) did not measure $\mathrm{N}$ abundances in their work. They did, however, measure the carbon isotopic ratio, finding ${ }^{12} \mathrm{C} /{ }^{13} \mathrm{C}=11$, which is very close to our best-fit value given in Table 3.

The final value of $[\mathrm{C} / \mathrm{Fe}]$ for the Li-rich giants ranges from -0.7 for $\mathrm{C} 1012254-203007$ and T9112-00430-1 to +0.3 for J043154.1-063210, while all stars have $[\mathrm{N} / \mathrm{Fe}]$ and $[\mathrm{O} / \mathrm{Fe}]$ values greater than zero. In Figure 5, we also plot the ratios found for the RAVE-VMP comparison sample described in Section 4.1. As illustrated in the figure, all Li-rich giants fall within the general trend of the RAVE-VMP stars. The $[\mathrm{C} / \mathrm{Fe}]$ and $[\mathrm{O} / \mathrm{Fe}]$ ratios appear to possibly increase with gravity, while the $[\mathrm{N} / \mathrm{Fe}]$ ratio may decrease with gravity. We computed Spearman's rank correlation coefficient, $r_{s}$, to investigate the level of correlation between all three ratios and $\log g$. We found that $[\mathrm{C} / \mathrm{Fe}]$ weakly correlates with gravity, with a value of $r_{s} \sim+0.5$. However, we found values of $r_{s} \sim+0.3$ and -0.3 for the $[\mathrm{O} / \mathrm{Fe}]$ and $[\mathrm{N} / \mathrm{Fe}]$ ratios, respectively, which implies no significant correlation. The weak trend in $[\mathrm{C} / \mathrm{Fe}]$ is a sign that the lower gravity stars have been affected by slightly more CNO cycling and internal mixing (see also Section 5). The 

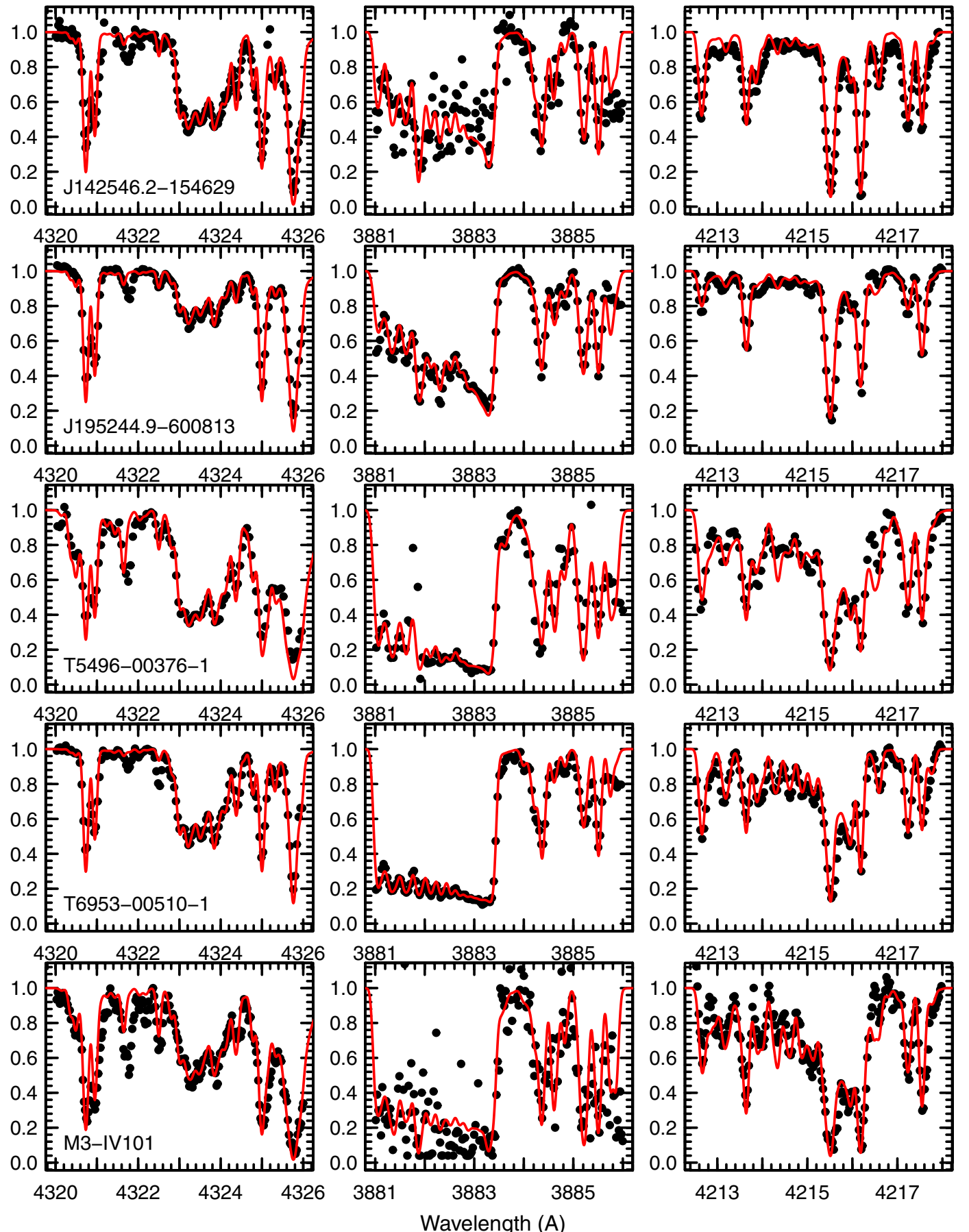

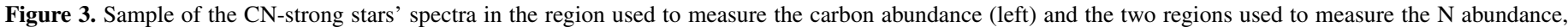
CN-3883 (middle) and CN-4216 (right). The solid red line represents the synthesis with the best-fit parameters given in Table 3 .

(A color version of this figure is available in the online journal.)

Li-rich giants at large gravity $(\log g>1.5)$ show the possibility of a slight enhancement in $[\mathrm{N} / \mathrm{Fe}]$ as compared to the RAVE-VMP stars, but this is not conclusive given the errors. We will discuss these trends in more detail for the entire RAVE-VMP sample in a later paper, but the main point to take away is that the Li-rich giants and RAVE-VMP stars appear to have experienced very similar $\mathrm{CNO}$ cycling.

All of the Li-rich giants have a ratio of the number of $\mathrm{C}$ atoms to $\mathrm{O}$ atoms, $\log (\mathrm{C} / \mathrm{O})<0$, which can be an indicator of the nature of their evolution should any of them be AGB stars (see Section 5.3 for more details). M3-IV101 and T6953-00510-1, however, have $\log (\mathrm{N} / \mathrm{O}) \gtrsim 0$. Further, like in [N/Fe], the Li-rich giants at large $\log g$ show indications of a slight enhancement in $\log (\mathrm{N} / \mathrm{O})$ as compared to the RAVE-VMP stars. Note that T6953-00510-1 is the most enhanced in $[\mathrm{N} / \mathrm{Fe}]$ and $[\mathrm{O} / \mathrm{Fe}]$ and J043154.1-063210 has the highest enhancement in $[\mathrm{C} / \mathrm{Fe}]$ among the Li-rich giants.

\subsection{Additional Elements}

We have used the line lists of Fulbright (2000) and Johnson (2002) to measure the abundances of other elements for these 

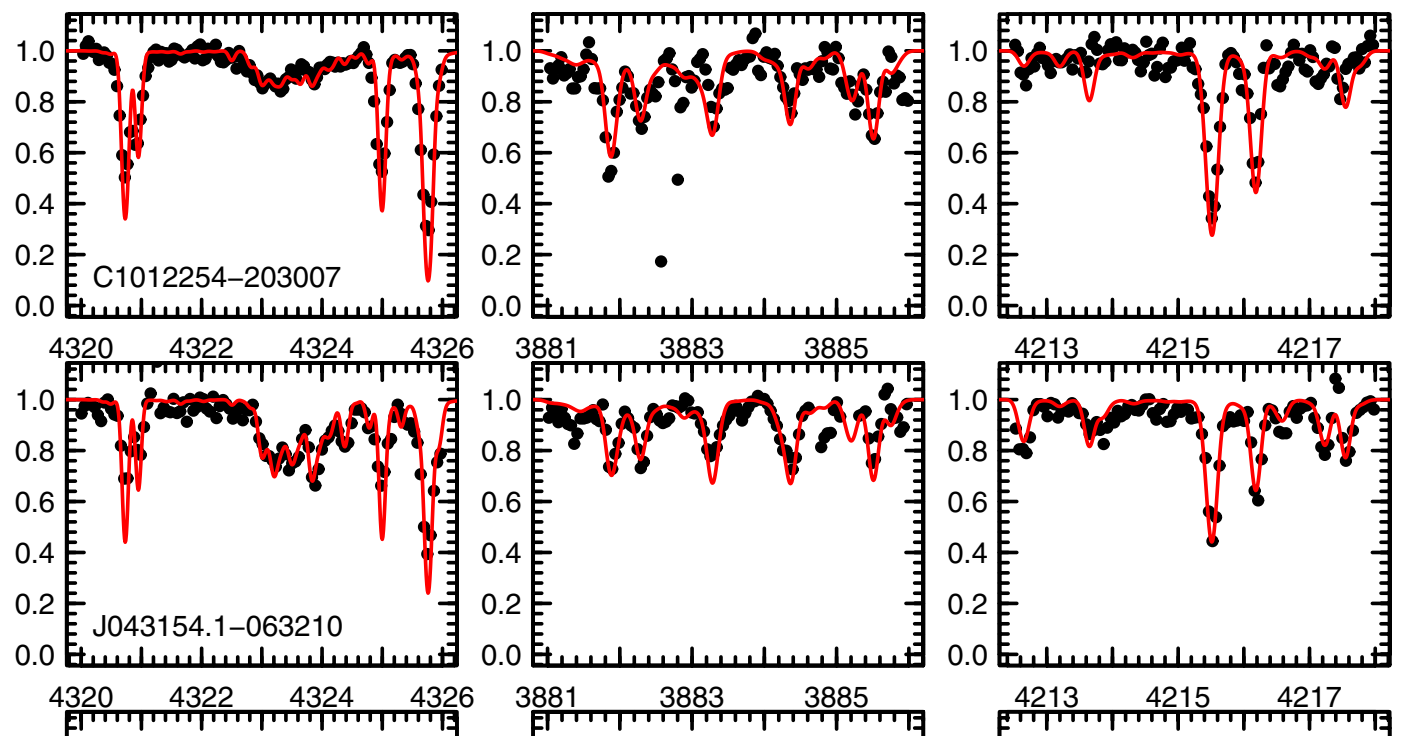

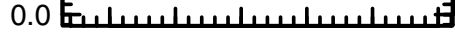
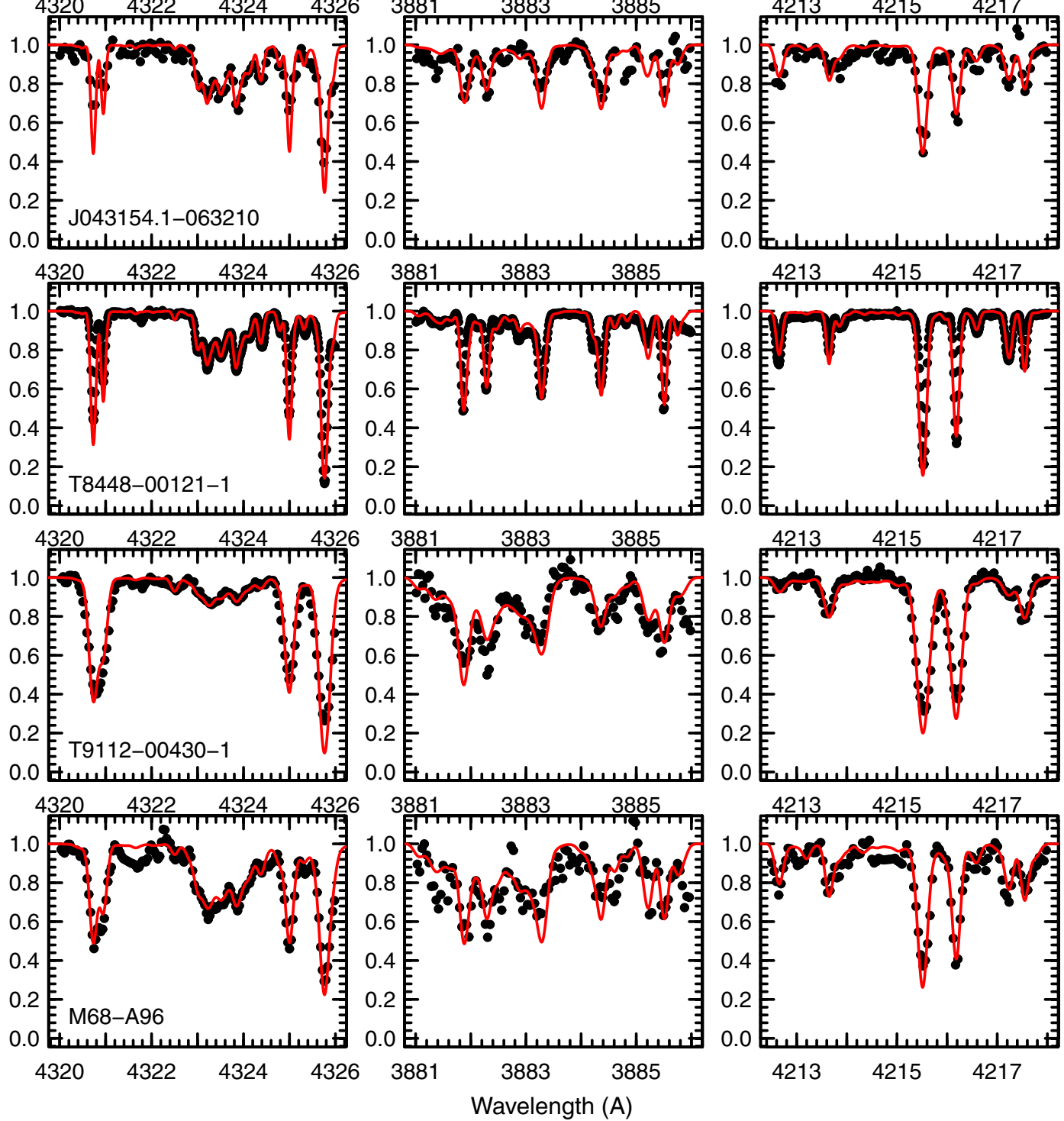

Figure 4. Same as Figure 3, but for those stars with a weak CN-3883 band.

(A color version of this figure is available in the online journal.)

stars, including $\mathrm{Mg}, \mathrm{Na}$, and several neutron-capture elements, for which the ratio with iron is given in Table 4. Hyperfine splitting effects were taken into account for the Na I D lines and the lines of Ba II and Eu II. Solar values for all elements were again selected from Asplund et al. (2009). The neutron-capture elements can be especially important since they can indicate the possible presence of dredge-up in AGB stars.

The derived abundance ratios for these elements are shown versus $[\mathrm{Fe} / \mathrm{H}]$ and $\log g$ in Figures 6 and 7, respectively, for our Li-rich candidates. We further plot the abundance ratios of our Li-normal VMP giants. Most of our stars have ratios that resemble that of the Li-normal VMP giants of like metallicity and gravity. The Li-rich giant T6953-00510-1, however, shows enhancement in the $s$-process elements ( $\mathrm{Sr}, \mathrm{Y}, \mathrm{Zr}, \mathrm{Ba}, \mathrm{La}, \mathrm{Pb}$ ) as compared to the other stars. Further evidence for $s$-process enhancement is found in the top plots of Figure 8, in which T6953-00510-1 shows enhancement in [Ba/Eu], which gives the ratio of $s$-process to $r$-process in a star. Other ratios, such as [Y/Zr], [Y/Ba], and [Ba/Eu] (see Figure 8), are similar between the Li-rich giants and Li-normal VMP stars.

Since T6953-00510-1 is the only star with enhanced $s$-process abundances, this enhancement is probably not connected to the mechanism that is enriching the $\mathrm{Li}$ in our stars. Further, this star is not located near the TP (thermally pulsing)-AGB evolutionary 
Table 4

Elemental Abundance Data

\begin{tabular}{|c|c|c|c|c|c|c|c|c|c|c|c|c|c|}
\hline Star & {$[\mathrm{Fe} / \mathrm{H}]$} & $\mathrm{Na}^{\mathrm{a}}$ & $\mathrm{Mg}^{\mathrm{a}}$ & $\mathrm{Sr}^{\mathrm{a}}$ & $\mathrm{Y}^{\mathrm{a}}$ & $\mathrm{Zr}^{\mathrm{a}}$ & $\mathrm{Ba}^{\mathrm{a}}$ & $\mathrm{La}^{\mathrm{a}}$ & $\mathrm{Eu}^{\mathrm{a}}$ & $\mathrm{Pb}^{\mathrm{a}}$ & {$[\mathrm{Ba} / \mathrm{Eu}]$} & {$[\mathrm{Y} / \mathrm{Zr}]$} & [Y/Ba] \\
\hline C1012254-203007 & -2.55 & - & 0.41 & 0.01 & -0.24 & 0.08 & 0.10 & 0.37 & 0.51 & - & -0.41 & -0.32 & -0.34 \\
\hline J043154.1-063210 & -1.85 & 0.08 & 0.46 & -0.01 & -0.29 & 0.24 & -0.50 & - & 0.70 & - & -1.20 & -0.53 & 0.21 \\
\hline J142546.2-154629 & -2.08 & -0.24 & 0.60 & -0.02 & -0.38 & 0.08 & -0.29 & 0.00 & 0.14 & - & -0.43 & -0.46 & -0.09 \\
\hline J195244.9-600813 & -1.41 & 0.14 & 0.40 & 0.02 & -0.08 & 0.32 & 0.13 & 0.18 & 0.29 & 0.19 & -0.16 & -0.40 & -0.21 \\
\hline T5496-00376-1 & -0.63 & 0.19 & 0.13 & -0.24 & -0.20 & -0.22 & 0.18 & 0.17 & 0.23 & 0.22 & -0.06 & 0.02 & -0.38 \\
\hline T6953-00510-1 & -1.93 & 0.21 & 0.33 & 0.17 & 0.13 & 0.51 & 0.89 & 1.26 & 0.33 & 1.48 & 0.56 & -0.38 & -0.76 \\
\hline T8448-00121-1 & -2.45 & -0.01 & 0.30 & - & -0.18 & - & -0.82 & - & - & - & - & - & 0.64 \\
\hline T9112-00430-1 & -2.21 & 0.00 & 0.45 & 0.19 & -0.40 & -0.12 & -0.30 & 0.28 & 0.71 & - & -1.01 & -0.28 & -0.10 \\
\hline M3-IV101 & -1.52 & 0.34 & 0.33 & 0.05 & 0.08 & 0.38 & 0.17 & 0.28 & -0.03 & 0.26 & 0.20 & -0.30 & -0.09 \\
\hline M68-A96 & -2.18 & 0.14 & 0.44 & -0.10 & -0.44 & 0.05 & -0.06 & 0.21 & 0.07 & 0.53 & -0.13 & -0.49 & -0.38 \\
\hline
\end{tabular}

Note. ${ }^{\text {a }}$ Given as $[X / \mathrm{Fe}]$.

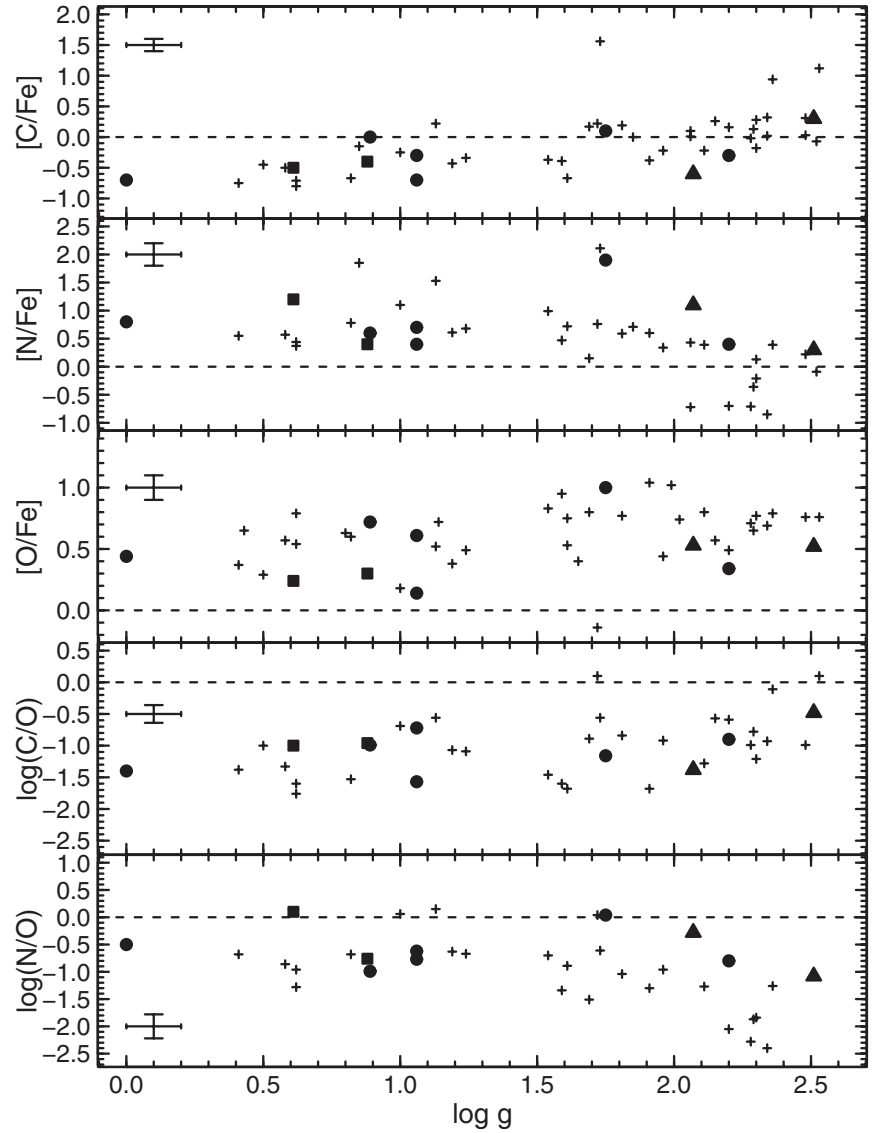

Figure 5. $\mathrm{CNO}$ abundance ratios with $\mathrm{Fe}$, as well as the log of the number ratios of $\mathrm{C} / \mathrm{O}$ and $\mathrm{N} / \mathrm{O}$, are shown vs. $\log g$. The six Li-rich field giants are represented as circles, while the two Li-normal field giants are shown as triangles. The two Li-rich globular cluster giants are shown as squares. The VMP comparison sample are shown as plus symbols. Note that all of the Li-rich giants have $\mathrm{C} / \mathrm{O}<1(\log (\mathrm{C} / \mathrm{O})<0)$, but two stars have $\mathrm{N} / \mathrm{O} \gtrsim 1$. T6953-00510-1 has the highest enhancement in $\mathrm{N}$ and $\mathrm{O}$, while J043154.1-063210 has the highest enhancement in $\mathrm{C}$.

phase (see Figure 1) where we would expect dredge-up of $s$-process enriched material. The simplest explanation is that this star is a part of a binary system in which its atmosphere has been polluted by a higher mass companion that has already gone through its TP-AGB phase. We do not detect any variation in the radial velocity of this star (see Section 4.5), but this scenario is still possible if the binary system is wide. It is also possible that T6953-00510-1 formed from $s$-process enriched material left by a star of an early generation that had gone through its TP-AGB
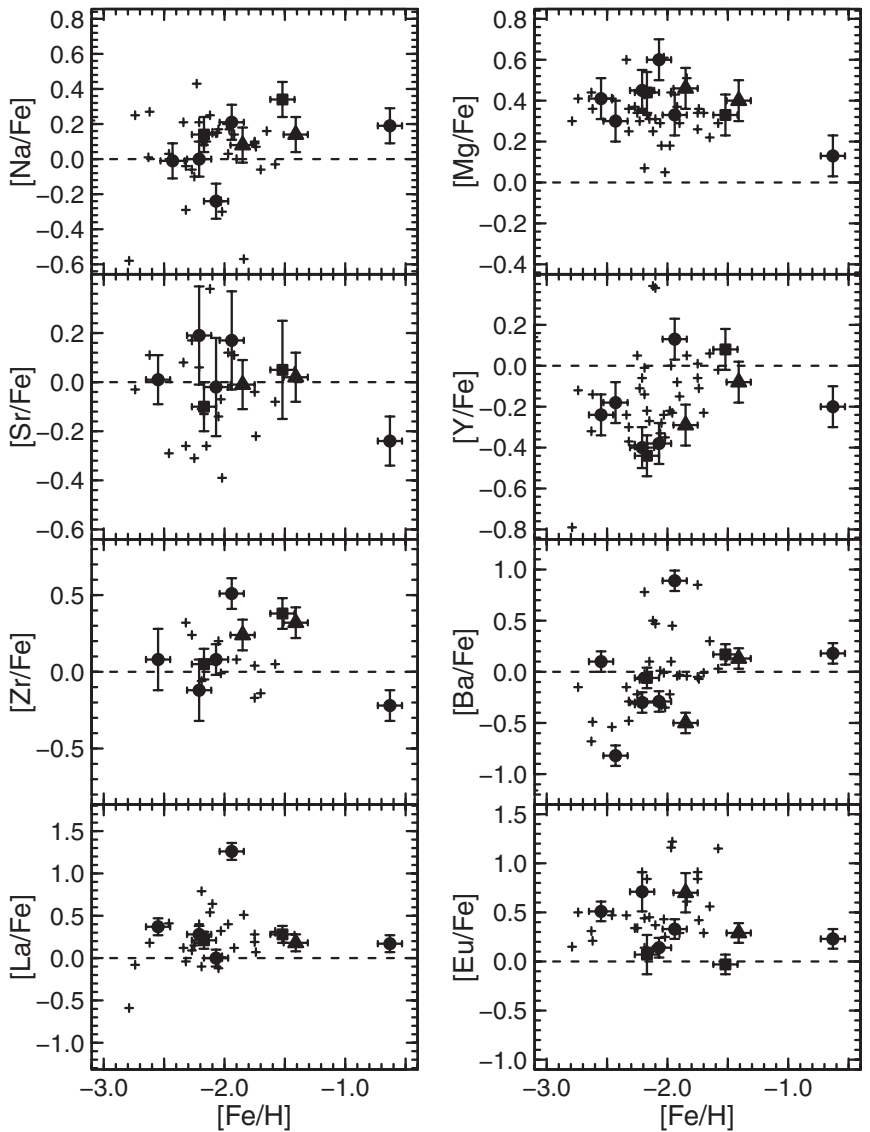

Figure 6. Ratio of several elements with iron abundance vs. [Fe/H]. The symbols are the same as in Figure 5. Pb is not shown since it could not be measured for several stars. Note the only Li-rich giant that shows consistent enhancement in the $s$-process (including $\mathrm{Pb}$ ) is T6953-00510-1.

phase, but this scenario would require extremely incomplete mixing of the interstellar medium prior to the next generation of stars.

Lee et al. (2005) measured abundances for seven stars in M68, but they did not include M68 A-96. If we use their line list and follow their analysis methods, we measure abundances for this star nearly identical to their mean cluster values.

\subsection{Projected Rotational Velocity}

Some have found that many of their metal-rich Li-rich giants had high projected rotational velocities, $v \sin i$ (e.g., Guillout et al. 2009). Further, Drake et al. (2002) suggested that the 

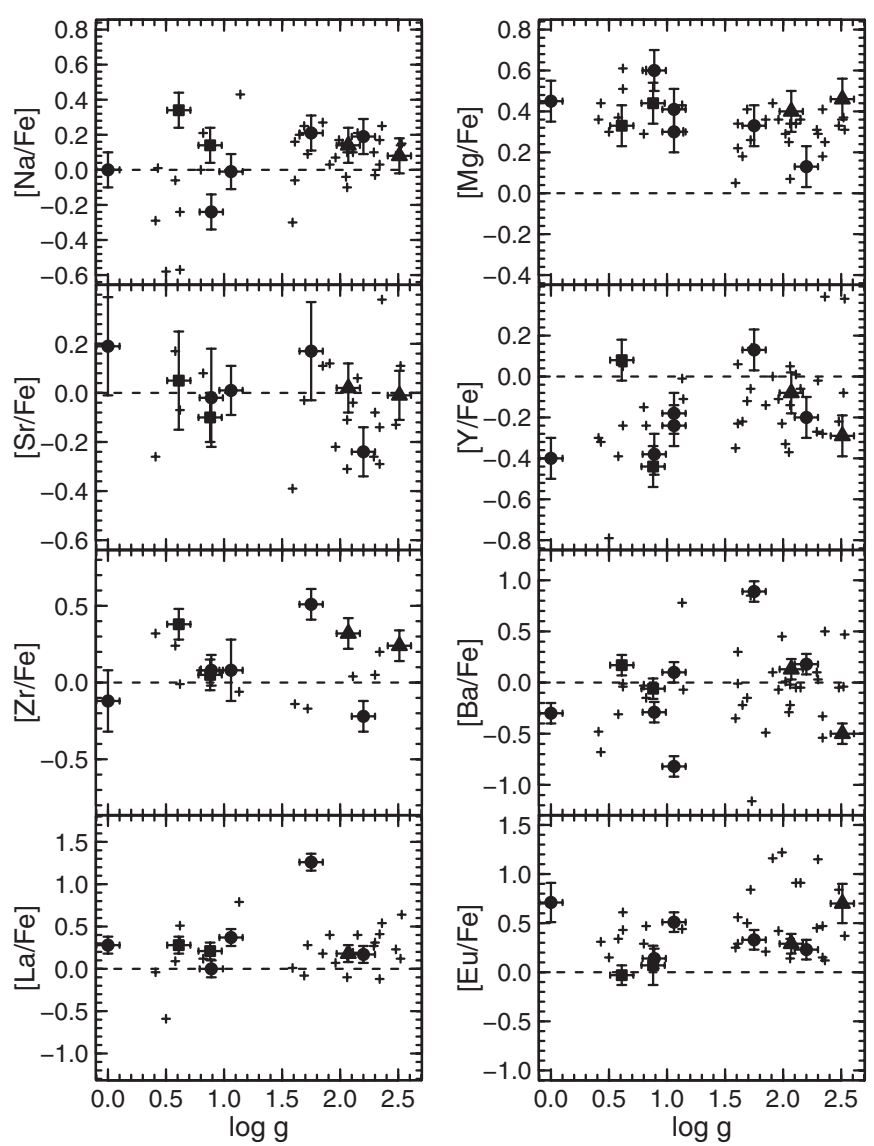

Figure 7. Ratio of several elements with iron abundance vs. $\log g$. The symbols are the same as in Figure 5. As in Figure 6, the Li-rich giants do not show any deviation from the general trends of the VMP comparison sample. Note that the apparent enhancement in [Y/Fe] of M3-IV101 is a metallicity artifact.

fraction of Li-rich stars can be as high as $50 \%$ among rapidly rotating giants. We therefore computed $v \sin i$ for the Li-rich giants in our sample to determine if any are rapid rotators. We derived $v \sin i$ following the methodology of Fekel (1997) and Hekker \& Meléndez (2007). First, the measured stellar broadening, $X_{\text {meas }}$, was estimated as the average of the full width at half-maximum (FWHM) of several Fe I lines near $6750 \AA$. The FWHM of several ThAr lines (from arc spectra taken during the night of each observation) in the same wavelength region as the Fe I lines were averaged to estimate the instrumental broadening, $X_{\text {inst }}$. The intrinsic broadening can then be estimated as

$$
X_{\text {intr }}=\sqrt{X_{\text {meas }}^{2}-X_{\text {inst }}^{2}} \text {. }
$$

We then determined the total broadening, $X_{\text {tot }}$, given our value of $X_{\text {intr }}$ for each star, using the second-order polynomial fit to $X_{\text {intr }}$ vs. $X_{\text {tot }}$ from Hekker \& Meléndez (2007):

$$
X_{\text {intr }}=0.10963+0.002758 X_{\text {tot }}+0.001278 X_{\text {tot }}^{2} .
$$

The projected rotational velocity, $v \sin i$, of a given star can then be computed as $\sqrt{X_{\mathrm{tot}}^{2}-v_{\mathrm{m}}^{2}}$, where $v_{m}$ is the macroturbulent velocity of that star. We adopted the relations between $v_{m}$ and $T_{\text {eff }}$ in Hekker \& Meléndez (2007) for different luminosity classes to estimate $v_{m}$ for each Li-rich giant. We assumed that the Li-rich giants in our sample near the RGB tip were class II and those near the RGB bump were class III. Note that those stars with estimated values of $v_{m}$ greater than the total broadening are assumed to have a non-measurable rotational velocity.

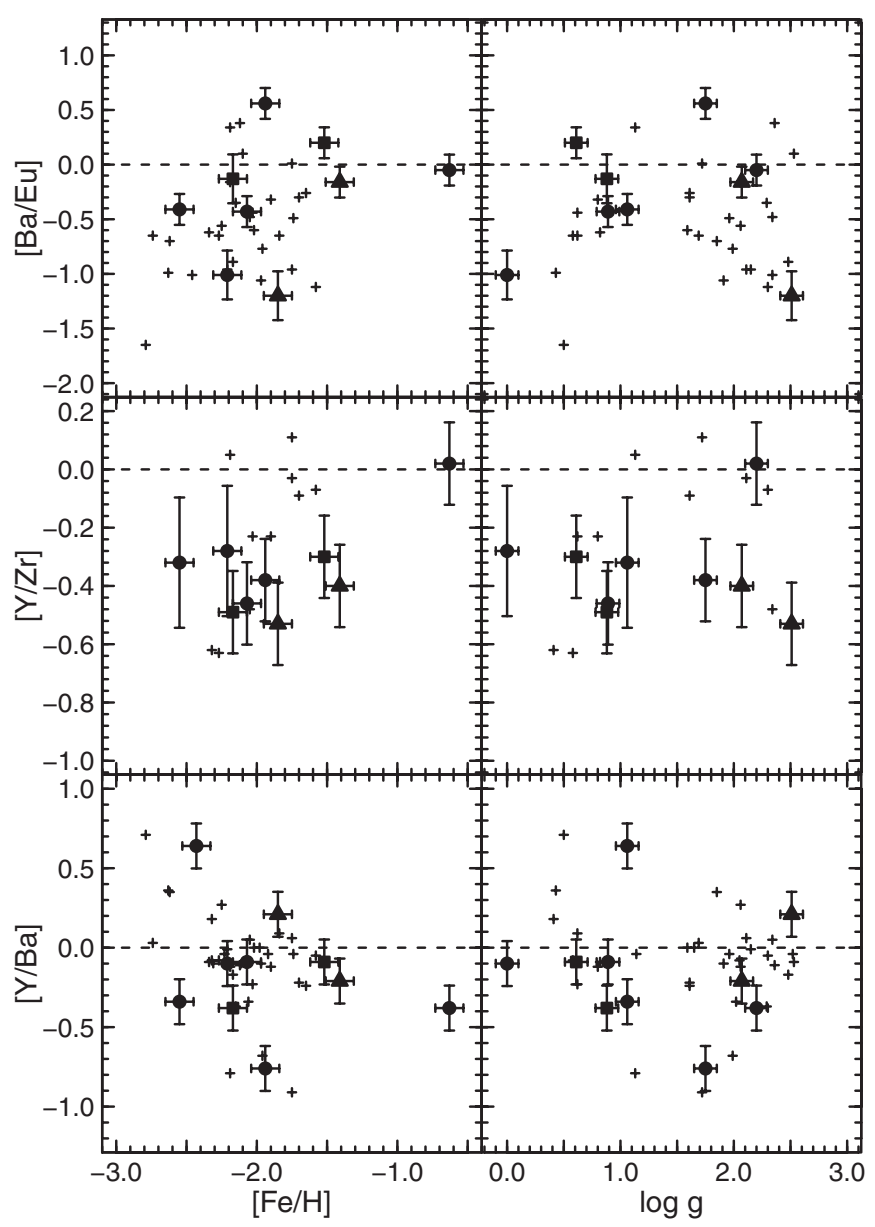

Figure 8. Ratio of several neutron-capture element abundances vs. metallicity. The symbols and lines are the same as in Figure 5. Note that T6953-00510-1 shows enhancement in $[\mathrm{Ba} / \mathrm{Eu}]$, suggesting (as in Figures 6 and 7) that it is $s$-process enhanced.

The majority of our Li-rich giants showed no measurable rotational velocity. We found non-negligible values of $\sim 3 \mathrm{~km} \mathrm{~s}^{-1}$ for T5496-00376-1 and $\sim 4 \mathrm{~km} \mathrm{~s}^{-1}$ for T8448-00121-1, but these values are only slightly larger than the expected projected rotation of low-mass giants, $v \sin i \leqslant 2 \mathrm{~km} \mathrm{~s}^{-1}$ (de Medeiros et al. 1996). C1012254-203007 and T9112-00430-1, however, have $v \sin i=8-10 \mathrm{~km} \mathrm{~s}^{-1}$. This implies that they very well could be rapidly rotating.

What would cause these stars to rapidly rotate? It is possible that extra angular momentum, dredged-up as mass is redistributed during increased convection, could induce increased rotation in these stars (Fekel \& Balachandran 1993). Another possibility is that these stars have accreted material from a planet or companion star, which would also contribute extra angular momentum to the stars. If indeed the two rapidly rotating stars are in a binary system, signatures may be present in their spectra; this is discussed below.

\subsection{Radial Velocity Variations}

None of the echelle data shows obvious line profile asymmetries or other spectral signs of having a bright secondary star in the system, but the light from a low-luminosity main-sequence or white dwarf companion would be swamped by the light from the bright giant star in the optical spectrum. 
For most of our stars, we only have two radial velocity measurements: the initial RAVE DR3 (Siebert et al. 2011) observation (with a systematic uncertainty of about $3 \mathrm{~km} \mathrm{~s}^{-1}$ ) and the echelle observation discussed here. RAVE observed two of the stars twice, and we have three echelle observations of C1012254-203007. All together, we have 21 radial velocity measurements of the eight RAVE Li-rich stars. The mean difference (RAVE-echelle) in the heliocentric radial velocities is $0.8 \pm 1.8 \mathrm{~km} \mathrm{~s}^{-1}$, which lies within the RAVE RV measurement error.

Both T9112-00430-1 and T6953-00510-1 shared the largest difference of $3.8 \mathrm{~km} \mathrm{~s}^{-1}$ and $3.4 \mathrm{~km} \mathrm{~s}^{-1}$, respectively, between two observations of the same star. These differences, however, are only $\sim 0.5-1.0 \mathrm{~km} \mathrm{~s}^{-1}$ larger than the systematic uncertainty of the RAVE radial velocities. All other repeat observations have a velocity difference of less than twice the internal uncertainties.

\subsection{Mass Loss}

We further searched the spectra of our candidate Li-rich giants for signs of possible mass loss, inspecting the strong photospheric lines in each spectrum, such as $\mathrm{H} \alpha$, the $\mathrm{Na} \mathrm{D}$ lines, and $\mathrm{Ca}$ II $\mathrm{H}$ and $\mathrm{K}$ lines (Balachandran et al. 2000; Drake et al. 2002). The only two stars in our sample to show possible evidence of mass loss are T9112-00430-1 and J142546.2154629. In both cases, we identified emission in the wings of $\mathrm{H} \alpha$. However, the emission was less pronounced in the spectrum of J142546.2-154629. Both stars showed no other signs (in the $\mathrm{Na} \mathrm{D}$ or $\mathrm{Ca} I \mathrm{H} \mathrm{H}$ and $\mathrm{K}$ lines) of mass loss in their spectra. The emission features could be an indicator that these stars are evolving on the AGB (see Section 5.3). Further, this mass loss could be a possible trigger for Li production in these stars (De la Reza et al. 1996, 2000); however, an investigation of infrared excess is needed to confirm this.

\section{LITHIUM PRODUCTION FROM RGB TO AGB}

The stage of evolution (AGB or RGB) in which each star belongs is an important aspect for understanding the mechanisms that underlie Li production. We therefore investigated the abundance patterns in each star in an attempt to find indicators for their evolutionary status, as well as Li production mechanism.

\subsection{Li Enrichment on the RGB}

Early on in a low-mass star's climb up the RGB, it undergoes the first dredge-up on the RGB after its outer convective envelope reaches the shell-burning regions. Standard theories of low-mass stellar evolution predict that the ${ }^{12} \mathrm{C} /{ }^{13} \mathrm{C}$ ratio drops from over 60 to about 40 around the point of the first dredge-up (Gratton et al. 2000). The first dredge-up mixes fresh $\mathrm{H}$ on the surface layers to the interior of the star, which causes a strong molecular weight discontinuity at the deepest extent of the convective envelope. It has been suggested that this molecular weight discontinuity, combined with rotation, induces extra mixing, such as thermohaline mixing, that triggers Li enrichment at the RGB bump (Charbonnel \& Balachandran 2000; Ulrich 1972; Kippenhahn et al. 1980; Charbonnel \& Zahn 2007; Eggleton et al. 2008). Further, Charbonnel \& Zahn (2007) found that for low-mass $\left(M<0.9 M_{\odot}\right)$ metal-poor $([\mathrm{Fe} / \mathrm{H}]<-0.5)$ stars, carbon and ${ }^{12} \mathrm{C} /{ }^{13} \mathrm{C}$ are also reduced while the nitrogen abundance increases. These trends predicted by thermohaline mixing were shown by Angelou et al. (2011) to agree with observational data in the study of the $\mathrm{CNO}$ abundances in M3 stars.

The two Li-normal giants (J043154.1-063210 and J195244.9600813) are most likely evolving along the RGB. J043154.1063210 lies at a higher gravity than the RGB luminosity bump (see Figure 1). Should it be on the RGB, it is most likely going through its first dredge-up. J195244.9-600813, on the other hand, appears to lie on the RGB bump. It is possible that both stars are at the beginning of the RGB-bump mixing phase. This would also explain the intermediate (best-fit) value of the ${ }^{12} \mathrm{C} /{ }^{13} \mathrm{C}$ ratio $(\sim 12)$ of J043154.1-063210 since the mixing has not yet reduced the ratio (Charbonnel \& Do Nascimento 1998; Gratton et al. 2004, and references therein).

The picture is more complicated, however, for our Li-rich giants. The majority of these stars have best-fit ${ }^{12} \mathrm{C} /{ }^{13} \mathrm{C}$ values greater than 10. This is unexpected beyond the RGB bump, given the above scenario, especially once a star has reached the RGB tip. One possibility is that our best-fit ${ }^{12} \mathrm{C} /{ }^{13} \mathrm{C}$ values are overestimated due to a flat likelihood peak. For example, T549600376-1, the most metal-rich $([\mathrm{Fe} / \mathrm{H}]=-0.63)$ giant in our sample, is the only Li-rich giant with stellar parameter values consistent with the RGB bump. The best-fit carbon isotopic ratio for this star, however, is a bit larger $(\sim 15)$ than expected if Li enrichment took place as described above. Our lower limit to ${ }^{12} \mathrm{C} /{ }^{13} \mathrm{C}$, however, is 5 , which is much closer to that predicted by Li enrichment at the RGB bump. Alternatively, this star may not have finished this mixing episode, which would imply that the ${ }^{12} \mathrm{C} /{ }^{13} \mathrm{C}$ ratio is still decreasing and that its $\mathrm{Li}$ abundance is still increasing. However, this solution would not explain those stars near the RGB tip that would have finished the Li-enrichment phase at the RGB bump.

The Li enrichment obtained at the RGB bump is also expected to decrease as a star evolves beyond the RGB bump due to normal Li dilution. Indeed, the Li-rich phase at the RGB bump is quite short. According to Denissenkov \& Herwig (2004), the phase should last no longer than a few million years. This implies that the Li should be (at least partially) depleted by the time a star reaches the RGB tip. According to the H-R diagram, T6953-00510-1 has a gravity and temperature consistent with it being on the RGB, but it has most likely evolved past the RGB bump. Its $\mathrm{Li}$ abundance is less than that of T5496-00376-1, as well as those found for solar metallicity stars (cf., Kumar et al. 2011). It could therefore have ended the extra mixing phase at the RGB bump and its $\mathrm{Li}$ abundance is now being depleted. Also note that it has a low carbon isotopic ratio, ${ }^{12} \mathrm{C} /{ }^{13} \mathrm{C} \sim 5$, suggesting that it has gone through thermohaline mixing.

It should be noted that this star is the only one in our sample that shows consistent $s$-process enhancement (as discussed in Section 4.3). We also argue that it may be "CNO-increased," in that its CNO abundances are somewhat enhanced in comparison to the other Li-rich stars in our sample. This star is not evolved enough to have produced (and dredged-up) the $s$-process and CNO abundances in its atmosphere. Most likely these enhancements are due to pre-enrichment at birth or mass transfer in a long-period binary. Although we found no conclusive disparities in the radial velocity of T6953-00510-1 (see Section 4.5), radial velocity variation from a long-period binary would be virtually undetectable without a dedicated radial velocity study.

The high-luminosity Li-rich giants in our sample, however, have $\mathrm{Li}$ abundances equal to or greater than the stars in our sample near the RGB bump (as well as that of solar-metallicity stars in previous samples), contrary to what was predicted for 
Li enrichment at the RGB bump (e.g., Charbonnel \& Primas 2005; Kumar et al. 2011). A solution to this discrepancy is that these stars have undergone $\mathrm{Li}$ enrichment via extra-deep mixing combined with CBP. Sackmann \& Boothroyd (1999) show that CBP can take place anywhere along the RGB, while the amount of $\mathrm{Li}$ enrichment depends strongly on the rate of mixing in the star. They further predict that the maximum Li abundance attained by a star can occur before significant amounts of ${ }^{13} \mathrm{C}$ lower the ${ }^{12} \mathrm{C} /{ }^{13} \mathrm{C}$ ratio. Thus, Li-rich giants could have values of ${ }^{12} \mathrm{C} /{ }^{13} \mathrm{C}$ ranging from $\sim 4$ up to $\sim 30$, which is consistent with the values of ${ }^{12} \mathrm{C} /{ }^{13} \mathrm{C}$ found for our Li-rich giants. Note also that the projected rotational velocities $\left(v \sin i=8-10 \mathrm{~km} \mathrm{~s}^{-1}\right)$ of $\mathrm{C} 1012254-203007$ and T9112-00430-1 may be large enough to induce the high extra mixing rates needed to achieve $\mathrm{Li}$ enhancement via CBP.

Another possibility is the so-called Li flash of Palacios et al. (2001). This model also predicts that the Li-rich phase begins at the RGB luminosity function bump when ${ }^{7} \mathrm{Be}$, transported from the interior, decays into ${ }^{7} \mathrm{Li}$ and burns in a Li-burning shell. This extra energy rapidly increases the star's luminosity and forces extra mixing, including the transport of more ${ }^{7} \mathrm{Be}$ (which decays into ${ }^{7} \mathrm{Li}$ ) to the surface. This mixing initially does not change the surface carbon isotope ratios, but the enhanced convection eventually reaches the depth where ${ }^{12} \mathrm{C}$ is converted to ${ }^{13} \mathrm{C}$ and the temperature is high enough to burn the freshly minted $\mathrm{Li}$ $\left({ }^{7} \mathrm{Li}(\mathrm{p}, \alpha) \alpha\right)$. Both the surface $\mathrm{Li}$ abundance and ${ }^{12} \mathrm{C} /{ }^{13} \mathrm{C}$ ratio are then lowered.

Pilachowski et al. (2003) analyzed the previously discovered Li-rich giant M3 IV-101 and found that the intermediate value of the star's ${ }^{12} \mathrm{C} /{ }^{13} \mathrm{C}$ ratio (for which we found a similar value) and its high luminosity were consistent with the Li-flash model. Our analysis shows that several of the Li-rich giants are much brighter than the RGB bump and indeed that these stars have an estimated luminosity that is about 15 times the RGBbump luminosity. The Li-flash model of Palacios et al. (2001) predicts a factor of $\sim 5.5$ increase in luminosity, but based on an assumed $1.5 M_{\odot}$ solar-metallicity star, rather than the lowermass VMP stars studied here. Further, recall that Denissenkov \& Herwig (2004) showed that canonical extra mixing cannot be responsible for the $\mathrm{Li}$ flash. However, if this scenario were possible in low-mass stars, then it could explain the luminous Li-rich giants in our sample, but cannot provide a solution for the less luminous giants.

\subsection{Li Enrichment on the Horizontal Branch}

Kumar et al. (2011) found that their stars close to the RGB bump were more likely associated with the theoretical position of the red clump, in which metal-rich (or young) stars have begun their core He-burning. Since it is highly unlikely that the Li-rich phase at the RGB bump would last until the red clump, they postulated that the Cameron-Fowler mechanism may also play a role during the He-core flash for stars with $M<2.25 \mathrm{M}_{\odot}$.

This relies on the fact that enough ${ }^{3} \mathrm{He}$ has survived mixing along the RGB (e.g., thermohaline mixing) for the Cameron-Fowler mechanism to take effect. They suggest that stars experiencing this mechanism would survive as Li-rich giants for about $1 \%$ of their horizontal branch lifetime, which corresponds to a few Myr. Our stellar parameter values for T5496-00376-1 show that it is also consistent with the horizontal branch (see Figure 1). It is therefore possible that T5496-00376-1 could actually be a horizontal branch (core He-burning) star that has undergone $\mathrm{Li}$ production at the He-core flash.

\subsection{Li Enrichment on the AGB}

The high-luminosity stars in our sample also appear to be consistent with the early TP-AGB phase of evolution. T911200430-1, for example, is most likely already evolving on the AGB. Its position far above the RGB tip (see Figure 1) suggests that the derived values of the stellar parameters are being affected by strong departures from hydrostatic equilibrium. More importantly, T9112-00430-1 has been found to be a variable star according to the All Sky Automated Survey (ASAS) Catalog of Variable Stars (Pojmanski 2002). This is further corroborated by the presence of emission in the wings of $\mathrm{H} \alpha$ in its spectrum (see Section 4.6), which suggests that it could be surrounded by a circumstellar envelope.

Hot bottom burning is not expected for our stars. They are metal-poor, which implies that they could be old, and so have masses low enough $\left(M \lesssim 1 M_{\odot}\right)$ that the convective envelope and H-burning shell are not connected. However, given that enough ${ }^{3} \mathrm{He}$ remains after the stars' ascent of the RGB, CBP (as described in Section 1) can occur on the AGB. Extra deep mixing mechanisms, similar to that of an RGB star (e.g., thermohaline mixing), in the radiative layer of a low-mass AGB star can connect the H-burning shell with the convective envelope, which will then drive the production of ${ }^{7} \mathrm{Li}$ by the Cameron-Fowler mechanism. It is possible, however, that the Li enrichment from CBP on the AGB might be less than that on the RGB since the amount of ${ }^{3} \mathrm{He}$ is expected to be depleted from the RGB. As noted in Section 5.1, C1012254-203007 and T9112-00430-1 have projected rotation velocities large enough that efficient mixing speeds could also be achieved to produce $\mathrm{Li}$ enhancement via CBP on the AGB.

During the TP-AGB phase, third dredge-up episodes enrich the envelope with $s$-process elements that are synthesized during the period between thermal pulses, when ${ }^{13} \mathrm{C}$ in the star's interior is burned and supplies neutrons (Straniero et al. 1995). Our high-luminosity Li-rich giants show, however, no signs of $s$-process enrichment. This implies that if they are AGB stars, they have not gone through enough third dredge-up episodes to drive up the $s$-process. Further, Girardi et al. (2010) found that a low-mass $\left(M_{\text {ini }} \lesssim 1.0 M_{\odot}\right)$ low-metallicity AGB star would remain O-rich $(\log (\mathrm{C} / \mathrm{O})<0)$ for its entire TP-AGB evolution, which is consistent with the $\mathrm{CNO}$ abundances found for the high-luminosity Li-rich giants in our sample.

We conclude that those stars in our sample that are evolving on the AGB (e.g., T9112-00430-1) should be (early) TP-AGB objects of low mass where very efficient CBP can occur, while the core is not massive enough to drive the third dredge-up.

\subsection{External Interactions}

Denissenkov \& Herwig (2004) suggest that the influence of a nearby companion (giant planet or star) could induce the extra mixing necessary to bring ${ }^{7} \mathrm{Be}$ up to the cooler surface where $\mathrm{Li}$ is produced, by the reaction ${ }^{7} \mathrm{Be}\left(e^{-}, v\right)^{7} \mathrm{Li}$, and does not suffer rapid destruction from proton captures. Indeed, Li-rich dwarf stars that show possible lithium pollution from a companion star have been discovered in globular clusters (see, e.g., Koch et al. 2011; Monaco et al. 2011a). In particular, Koch et al. (2011) found a Li-rich star that showed no enrichment in $s$-process to be in a binary system. They suggested that the Li enrichment arose from mass transfer from a companion giant star which had undergone CBP.

The high projected rotational velocity found for C1012254203007 and T9112-00430-1 could have been produced by 
the transfer of angular momentum from a binary companion. While we did not have the observations to detect extrasolar planets around our stars, we checked the Schneider et al. (2011) extrasolar planet database but did not find coincidences. We further looked for radial velocity variations that might indicate a low-luminosity stellar-mass companion. The lack of large radial velocity variations over the whole sample strongly suggests that a close stellar-mass companion is not required for the Li-rich phase to occur. The abundances of T6953-00510-1, however, suggest that it may have been enriched through mass transfer by an evolved stellar companion in a wide binary. For this reason, recent $\mathrm{Li}$ enrichment (so that the $\mathrm{Li}$ has not yet burned away) from an evolved companion cannot be ruled out for this star.

\section{CONCLUSION}

This work presents the largest sample of metal-poor Li-rich giants to date. We have discovered five new metal-poor $([\mathrm{Fe} / \mathrm{H}] \lesssim-1.9)$ Li-rich giants, and one Li-rich giant at a larger metallicity of $\sim-0.6$ dex in the RAVE survey. These stars were found in a total sample of $\sim 700$ stars. This is consistent with the previous finding that about $1 \%$ of all giants are Li-rich, which suggests that the frequency of Li-rich giants is independent of metallicity.

We have further analyzed the newly discovered (in this work) Li-rich member of the globular cluster M68 and confirmed the large Li abundance of M3-IV101, whose Li-rich nature was discovered by Kraft et al. (1999). The Li-rich giant in M68 adds to the very small number of giants in globular clusters identified to have $\mathrm{Li}$ enrichment. Indeed, Pilachowski et al. (2000) did not find any Li-rich giants in a sample of over 200 giants selected from several globular clusters. Further, all Li-rich giants identified in globular clusters (including the giant in M68) have been found to be evolving near the RGB tip.

We performed a detailed abundance analysis of all stars and found that, aside from $\mathrm{Li}$, the majority of the Li-rich giants in our sample have abundance trends that resemble that of the RAVE-VMP comparison sample. This is consistent with, and extends, the findings of Castilho et al. (2000) for solarmetallicity Li-rich giants. The Li-rich giants in our sample are relatively carbon-poor and nitrogen-rich, with normal oxygen abundances found for metal-poor halo giants. Only one star in our sample, namely T6953-00510-1, shows enhancements in C, $\mathrm{N}$, and the $s$-process elements. We attribute these enhancements to either pre-enrichment or binary pollution and conclude that such enhancements are not connected to the mechanisms that produce Li in our full sample. Although we found no large radial velocity variations for the stars in our sample, a radial velocity study, over a longer time period, would be useful to determine if any of our giants belong to a long-period binary. We further computed the projected rotational velocities of our stars, finding that only two stars, C1012254-203007 and T9112-00430-1, are rapidly rotating with $v \sin i>8 \mathrm{~km} \mathrm{~s}^{-1}$.

An important finding is that the high-luminosity giants can have $\mathrm{Li}$ abundances equal to those found for giants near the RGB bump, which has also been found for stars in other environments and higher metallicities (Domínguez et al. 2004; Kraft et al. 1999; Monaco \& Bonifacio 2008; Monaco et al. 2011b). If the stars at the RGB bump and RGB tip were enriched in Li by the same process, this would argue against a single Li-enrichment phase at the RGB bump. In this case, the RGB-tip giants should have lower Li abundances than those at the RGB bump, contrary to our results. Instead, the most likely scenario is that the
Li-rich giants have undergone $\mathrm{Li}$ enrichment via cool bottom processing. This process is also in agreement with our best-fit ${ }^{12} \mathrm{C} /{ }^{13} \mathrm{C}$ values for the Li-rich giants. Further, the metallicity and Galactic population membership (e.g., thick disk and halo) of the Li-rich giants in our sample are consistent with old ages and low masses $\left(<1 M_{\odot}\right)$. Our identification of luminous Lirich giants evolving above the RGB bump therefore contrasts with the Charbonnel \& Balachandran (2000) suggestion that Li-rich giants should be found above the RGB bump only in intermediate-mass stars.

It is possible that some of the luminous giants in our sample are AGB stars. In those cases, cool bottom processing is still feasible as long as some ${ }^{3} \mathrm{He}$ has survived after the RGB. Hot bottom burning is not possible for our stars, as this process requires a much larger stellar mass. We also cannot rule out the possibility that T5496-00376-1 is a horizontal branch star that was enriched in Li during its He-flash phase, but this has not been modeled. Future infrared observations of these stars would be beneficial for measuring any infrared excess that would imply that they are evolving on the AGB. In addition, asteroseismology observations obtained with the Kepler spacecraft could also be used to distinguish between RGB and HB stars (e.g., Bedding et al. 2011).

It is clear from this study that metal-poor Li-rich giants are crucial for constraining the models of Li production in giants. The discovery and analysis of more metal-poor Li-rich giants will allow for more robust statistics and enhance our understanding of these rare and important objects.

We thank the staff members of Siding Spring Observatory, La Silla Observatory, Apache Point Observatory, and Las Campanas Observatory for their assistance in making these observations possible. G.R.R. wishes to thank Karin Lind, Luca Sbordone, and Peter Cottrell for their useful discussions and suggestions. G.R.R. further thanks Bertrand Plez for kindly providing his molecular line lists and Luca Casagrande for providing his photometric temperatures. G.R.R. and R.F.G.W. acknowledge support from the NSF of the USA (AST-0908326). J.P.F. wishes to thank Jennifer Johnson and Howard Bond for their useful suggestions, and acknowledges support through grants from the W. M. Keck Foundation and the Gordon and Betty Moore Foundation, to establish a program of data-intensive science at the Johns Hopkins University. E.K.G. was partially supported by the Sonderforschungsbereich "The Milky Way System" (SFB 881, subproject A5) of the German Research Foundation (DFG). This publication makes use of data products of the Two Micron All Sky Survey, which is a joint project of the University of Massachusetts and IPAC/Caltech, funded by NASA and the NSF. This research has also made use of the Vizie-R databases, operated at CDS, Strasbourg, France. Funding for RAVE has been provided by the Australian Astronomical Observatory; the Leibniz-Institut für Astrophysik Potsdam (AIP); the Australian National University; the Australian Research Council; the French National Research Agency; the German Research Foundation; the European Research Council (ERC-StG 240271 Galactica); the Istituto Nazionale di Astrofisica at Padova; The Johns Hopkins University; the National Science Foundation of the USA (AST-0908326); the W. M. Keck foundation; the Macquarie University; the Netherlands Research School for Astronomy; the Natural Sciences and Engineering Research Council of Canada; the Slovenian Research Agency; the Swiss National Science Foundation; the Science \& Technology Facilities Council of the UK; Opticon; Strasbourg Observatory; and the 
Universities of Groningen, Heidelberg, and Sydney. The RAVE Web site is at http://www.rave-survey.org.

Facilities: ARC (echelle spectrograph), AAT (UCLES), Magellan:Clay (MIKE), Max Planck:2.2m (FEROS), UKST (6dF spectrograph)

\section{REFERENCES}

Alcaino, G. 1977, A\&AS, 29, 9

Allende Prieto, C., García López, R. J., Lambert, D. L., \& Gustafsson, B. 1999, ApJ, 527, 879

Angelou, G. C., Church, R. P., Stancliffe, R. J., Lattanzio, J. C., \& Smith, G. H. 2011, ApJ, 728, 79

Aoki, W., Barklem, P. S., Beers, T. C., et al. 2009, ApJ, 698, 1803

Asplund, M., Grevesse, N., Sauval, A. J., \& Scott, P. 2009, ARA\&A, 47, 481

Asplund, M., Lambert, D. L., Nissen, P. E., Primas, F., \& Smith, V. V. 2006, ApJ, 644, 229

Balachandran, S. C., Fekel, F. C., Henry, G. W., \& Uitenbroek, H. 2000, ApJ, 542,978

Bedding, T. R., Mosser, B., Huber, D., et al. 2011, Nature, 471, 608

Bonifacio, P., Molaro, P., Sivarani, T., et al. 2007, A\&A, 462, 851

Boothroyd, A. I., \& Sackmann, I.-J. 1999, ApJ, 510, 232

Boothroyd, A. I., Sackmann, I.-J., \& Wasserburg, G. J. 1995, ApJ, 442, L21

Brown, J. A., Sneden, C., Lambert, D. L., \& Dutchover, E., Jr. 1989, ApJS, 71, 293

Cameron, A. G. W. 1955, ApJ, 121, 144

Cameron, A. G. W., \& Fowler, W. A. 1971, ApJ, 164, 111

Carney, B. W., Fry, A. M., \& Gonzalez, G. 1998, AJ, 116, 2984

Castilho, B. V., Gregorio-Hetem, J., Spite, F., Barbuy, B., \& Spite, M. 2000, A\&A, 364, 674

Charbonnel, C. 1995, ApJ, 453, L41

Charbonnel, C., \& Balachandran, S. C. 2000, A\&A, 359, 563

Charbonnel, C., \& Do Nascimento, J. D., Jr. 1998, A\&A, 336, 915

Charbonnel, C., \& Primas, F. 2005, A\&A, 442, 961

Charbonnel, C., \& Zahn, J.-P. 2007, A\&A, 476, L29

Cyburt, R. H., Fields, B. D., \& Olive, K. A. 2008, J. Cosmol. Astropart. Phys., JCAP11(2008) 12

De la Reza, R., Da Silva, L., Drake, N. A., \& Terra, M. A. 2000, ApJ, 535, L115

De la Reza, R., Drake, N. A., \& Da Silva, L. 1996, ApJ, 456, L115

de Medeiros, J. R., Da Rocha, C., \& Mayor, M. 1996, A\&A, 314, 499

Denissenkov, P. A., \& Herwig, F. 2004, ApJ, 612, 1081

Denissenkov, P. A., Pinsonneault, M., \& MacGregor, K. B. 2009, ApJ, 696, 1823

Domínguez, I., Abia, C., Straniero, O., Cristallo, S., \& Pavlenko, Y. V. 2004, A\&A, 422, 1045

Drake, N. A., de la Reza, R., da Silva, L., \& Lambert, D. L. 2002, AJ, 123, 2703

Dunkley, J., Komatsu, E., Nolta, M. R., et al. 2009, ApJS, 180, 306

Eggleton, P. P., Dearborn, D. S. P., \& Lattanzio, J. C. 2008, ApJ, 677, 581

Fekel, F. C. 1997, PASP, 109, 514

Fekel, F. C., \& Balachandran, S. 1993, ApJ, 403, 708

Fekel, F. C., \& Watson, L. C. 1998, AJ, 116, 2466

Forestini, M., \& Charbonnel, C. 1997, A\&AS, 123, 241

Fulbright, J. P. 2000, AJ, 120, 1841 (F00)

Fulbright, J. P., \& Johnson, J. A. 2003, ApJ, 595, 1154

Fulbright, J. P., Wyse, R. F. G., Ruchti, G. R., et al. 2010, ApJ, 724, L104

Girardi, L., Williams, B. F., Gilbert, K. M., et al. 2010, ApJ, 724, 1030

Gonzalez, O. A., Zoccali, M., Monaco, L., et al. 2009, A\&A, 508, 289

González Hernández, J. I., \& Bonifacio, P. 2009, A\&A, 497, 497
Gratton, R., Sneden, C., \& Carretta, E. 2004, ARA\&A, 42, 385

Gratton, R. G., \& Sneden, C. 1990, A\&A, 234, 366

Gratton, R. G., Sneden, C., Carretta, E., \& Bragaglia, A. 2000, A\&A, 354, 169

Guillout, P., Klutsch, A., Frasca, A., et al. 2009, A\&A, 504, 829

Gustafsson, B., Edvardsson, B., Eriksson, K., et al. 2008, A\&A, 486, 951

Hekker, S., \& Meléndez, J. 2007, A\&A, 475, 100

Hill, V., Plez, B., Cayrel, R., et al. 2002, A\&A, 387, 560

Hosford, A., Ryan, S. G., García Pérez, A. E., Norris, J. E., \& Olive, K. A. 2009, A\&A, 493, 601

Huber, K. P., \& Herzberg, G. 1979, Molecular Spectra and Molecular Structure IV: Constants of Diatomic Molecules (New York, NY: van Nostrand Reinhold)

Jasniewicz, G., Parthasarathy, M., de Laverny, P., \& Thévenin, F. 1999, A\&A, 342,831

Johnson, H. L., \& Sandage, A. R. 1956, ApJ, 124, 379

Johnson, J. A. 2002, ApJS, 139, 219

Kippenhahn, R., Ruschenplatt, G., \& Thomas, H.-C. 1980, A\&A, 91, 175

Koch, A., Lind, K., \& Rich, R. M. 2011, ApJ, 738, L29

Kraft, R. P., \& Ivans, I. I. 2003, PASP, 115, 143

Kraft, R. P., Peterson, R. C., Guhathakurta, P., et al. 1999, ApJ, 518, L53

Kumar, Y. B., \& Reddy, B. E. 2009, ApJ, 703, L46

Kumar, Y. B., Reddy, B. E., \& Lambert, D. L. 2011, ApJ, 730, L12

Kupka, F. G., Ryabchikova, T. A., Piskunov, N. E., Stempels, H. C., \& Weiss, W. W. 2000, Balt. Astron., 9, 590

Lambert, D. L. 1978, MNRAS, 182, 249

Lee, J.-W., Carney, B. W., \& Habgood, M. J. 2005, AJ, 129, 251

Lind, K., Asplund, M., \& Barklem, P. S. 2009a, A\&A, 503, 541

Lind, K., Primas, F., Charbonnel, C., Grundahl, F., \& Asplund, M. 2009b, A\&A, 503,545

Marigo, P., Girardi, L., Bressan, A., et al. 2008, A\&A, 482, 883

Meléndez, J., \& Ramírez, I. 2004, ApJ, 615, L33

Monaco, L., \& Bonifacio, P. 2008, Mem. Soc. Astron. Ital., 79, 524

Monaco, L., Villanova, S., Bonifacio, P., et al. 2011a, arXiv:1108.0138

Monaco, L., Villanova, S., Moni Bidin, C., et al. 2011b, A\&A, 529, A90

Nollett, K. M., Busso, M., \& Wasserburg, G. J. 2003, ApJ, 582, 1036

Palacios, A., Charbonnel, C., \& Forestini, M. 2001, A\&A, 375, L9

Palacios, A., Charbonnel, C., Talon, S., \& Siess, L. 2006, A\&A, 453, 261

Pilachowski, C. A., Sneden, C., \& Booth, J. 1993, ApJ, 407, 713

Pilachowski, C. A., Sneden, C., Freeland, E., \& Caperson, J. 2003, AJ, 125, 794

Pilachowski, C. A., Sneden, C., Kraft, R. P., Harmer, D., \& Willmarth, D. 2000, AJ, 119, 2895

Pojmanski, G. 2002, Acta Astron., 52, 397

Roederer, I. U., Frebel, A., Shetrone, M. D., et al. 2008, ApJ, 679, 1549

Ruchti, G. R., Fulbright, J. P., Wyse, R. F. G., et al. 2010, ApJ, 721, L92

Ruchti, G. R., Fulbright, J. P., Wyse, R. F. G., et al. 2011, ApJ, 737, 9

Ryan, S. G., Kajino, T., Beers, T. C., et al. 2001, ApJ, 549, 55

Sackmann, I.-J., \& Boothroyd, A. I. 1999, ApJ, 510, 21

Salaris, M., Chieffi, A., \& Straniero, O. 1993, ApJ, 414, 580

Sbordone, L., Bonifacio, P., Caffau, E., et al. 2010, A\&A, 522, A26

Schneider, J., Dedieu, C., Le Sidaner, P., Savalle, R., \& Zolotukhin, I. 2011, A\&A, 532, A79

Siebert, A., Williams, M. E. K., Siviero, A., et al. 2011, AJ, 141, 187

Smith, V. V., Shetrone, M. D., \& Keane, M. J. 1999, ApJ, 516, L73

Sneden, C. 1973, ApJ, 184, 839

Spite, M., \& Spite, F. 1982a, Nature, 297, 483

Spite, F., \& Spite, M. 1982b, A\&A, 115, 357

Steigman, G. 2007, Annu. Rev. Nucl. Part. Sci., 57, 463

Steinmetz, M., Zwitter, T., Siebert, A., et al. 2006, AJ, 132, 1645

Straniero, O., Gallino, R., Busso, M., et al. 1995, ApJ, 440, L85

Thevenin, F., \& Idiart, T. P. 1999, ApJ, 521, 753

Ulrich, R. K. 1972, ApJ, 172, 165

Uttenthaler, S., Lebzelter, T., Palmerini, S., et al. 2007, A\&A, 471, L41 